\title{
Reduced basis method for multi-parameter dependent steady Navier-Stokes equations: applications to natural convection in a cavity
}

\author{
Simone Deparis, Gianluigi Rozza* \\ Ecole Polytechnique Fédérale de Lausanne, Modelling and Scientific Computing, \\ Station 8-MA, CH-1015, Lausanne, Switzerland
}

\begin{abstract}
This work focuses on the approximation of parametric steady Navier-Stokes equations by the reduced basis method. For a particular instance of the parameters under consideration, we are able to solve the underlying partial differential equations, compute an output, and give sharp error bounds. The computations are split into an offline part, where the value of the parameters is not yet identified, but only within a range of interest, and an online part, where the problem is solved for an instance of the parameters. The offline part is expensive and is used to build a reduced basis and prepare all the ingredients - mainly matrix-vector and scalar products, but also eigenvalue computations - necessary for the online part, which is fast.

We provide a model problem - describing natural convection phenomena in a laterally heated cavity — characterized by three parameters: Grashof and Prandtl numbers and the aspect ratio of the cavity. We show the feasibility and efficiency of the $a$ posteriori error estimation by the natural norm approach considering several test cases by varying two different parameters. The gain in terms of CPU time with respect to a parallel finite element approximation is of three magnitude orders with an acceptable — indeed less than $0.1 \%$ - error on the selected outputs.
\end{abstract}

Key words: Reduced basis method, a posteriori error estimation,

Brezzi-Rappaz-Raviart theory, inf-sup constant, steady incompressible

Navier-Stokes equations, natural convection, Prandtl number, Grashof number.

* Corresponding Author

Email address: simone.deparis@epfl.ch, gianluigi.rozza@epfl.ch

(Simone Deparis, Gianluigi Rozza). 


\section{Introduction and Motivation}

The reduced basis approximation (see $[23,30,21]$ ) is a discretization method for the solution of parametrized partial differential equations. It permits rapid and reliable evaluation of input-output relationship in the limit of many queries - in the design, optimization, control, and characterization contexts. We describe here a non-linear example: the case of the steady incompressible Navier-Stokes equations to model natural heat convection with more than one (physical, geometrical) parameter in affine dependence ${ }^{1}$. The case with one (physical) parameter within an affine parametric dependence has been treated in detail in [3] considering high Grashof numbers $\left(\sim 10^{7}\right)$ and using a natural norm approach, and, previously, in $[20,36]$ for lower Grashof numbers $\left(\sim 10^{4}\right)$. A field of interest for this kind of applications deals with microfluidics, in biomedical sciences, environmental sciences and, more generally, with mechanical engineering (automotive and aerospace industry).

The use of the reduced basis method in numerical fluid dynamics is aimed at providing real-time solutions and information on fluid mechanics outputs. Its extension to steady Navier-Stokes equations, which requires treatment of nonlinearities, provides, e.g., an efficient optimization toolbox in design problems with a certain degree of complexity. The study of parametrized systems is well suited also to carry out shape optimization and shape design problems considering several geometrical parameters.

The reduced basis approach and associated offline-online procedures can be applied without serious computational difficulties to quadratic nonlinearities. Much work focuses on the stationary incompressible (quadratically nonlinear) Navier-Stokes equations $[6,8,10]$ : suitable stable approximations were first considered in $[10,12,22]$, and more recently $[24,28]$ also for non-affine parametric dependence; rigorous a posteriori error estimation - within the general Brezzi-Rappaz-Raviart ("BRR") a posteriori framework $[1,2]$ - is considered in $[3,20,36,37]$. The latter is admittedly quite complicated, and presently limited to few parameters - Reynolds, Prandtl, Grashof numbers or an aspect ratio, as an example of geometrical parametrization. In this work we follow this line and focus our attention on the following aspects: i) the efficient treatment of the non-linear term; ii) the geometrical and physical parametrization; iii) the incorporation of a stable approximation for pressure [31]; iv) an accurate and feasible a posteriori error estimation based on the natural norm approach [3].

The aim of this paper is to provide extensive tests to validate and generalize the reduce basis method for natural convection problems in a rectangular cavity

\footnotetext{
$\overline{1}$ Affine dependence in defined by equation (4) and roughly means that the parameters enter in the weak form as a coefficient in front of the integrals.
} 
(see Figure 1) increasing the number of physical and geometrical parameters: in addition to the Grashof number (Gr), used in [3], we consider, the Prandtl number $(\operatorname{Pr})$ and the aspect ratio $(A)$ of the laterally heated cavity, combined only in couples such as $\mathrm{Gr}-\mathrm{Pr}, \mathrm{Gr}-A, \operatorname{Pr}-A$. The combination of physical and geometrical parameters in the same problem provides a wide variety of applications involving thermo-fluid-dynamics.

An additional effort has been devoted in testing all the ingredients we need to compute error bounds and a posteriori error estimation (as certificate of fidelity of the methodology) in the multi-parametric case: in particular the lower bound to the inf-sup constant and the eigenvalues problems involved [13]. In the end, given a parameter value, either we are able to give an explicit correct error bound, or we can not ensure existence or uniqueness (which means that we have to enrich our basis).

The present work is organized as follows: after this introduction, as a short review on reduced basis for Navier-Stokes equations, in Section 2.1 the natural convection problem is presented (see Figure 1). In Section 2.3 we recall the reduced basis formulation for Navier-Stokes equations, then in Sections 2.4, 2.5 and 2.6 we recall all the principal ingredients for the a posteriori error estimation based on natural norm and existence and uniqueness results. The stabilization of the reduced basis and the offline and online algorithms are considered in Section 3, with references to the literature for technicalities $[31,21,30]$. In Section 4 numerical results and computational costs and savings are reported for three different parameter combinations. Some conclusions and description of future works follow in Section 5.

\subsection{Offline-Online computational decomposition}

One of the keys in the reduced basis method is the decomposition of the computational work into an offline and an online stage (see $[30,21])$.

The former is carried out independently from a specific parameter of a problem at hand. A greedy algorithm is performed to search for the parameters that provide an optimized reduced basis for the Galerkin approximation. In the meantime, the ingredients for the resolution of the reduced discrete system and for the computation of the dual norm of the residual are computed. These hang on finite element matrix-vector and vector-vector products. In a second stage the error bound ingredients are built by solving a set of generalized eigenvalue problems.

The online stage involves a (some) particular instance(s) of the parameter. The system that has to be solved is the Galerkin projection on the space spanned by the reduced basis. The complexity of both the resolution of this system and 
the computation of the error bounds depends on the chosen number of basis but is independent from the number of degrees of freedom of the underlying finite element problem.

\subsection{Abstract formulation}

We are interested in the numerical approximation of parameter $(\boldsymbol{\mu})$ dependent nonlinear partial differential equations and the prediction of an "output of interest" which is a functional of the field variable $\boldsymbol{u}^{\mathrm{e}}(\boldsymbol{\mu})$,

$$
s^{\mathrm{e}}(\boldsymbol{\mu})=\ell\left(\boldsymbol{u}^{\mathrm{e}}(\boldsymbol{\mu})\right) \in \mathbb{R}
$$

where $\ell$ is a continuous linear form. The solution $\boldsymbol{u}^{\mathrm{e}}(\boldsymbol{\mu})$ may not be unique, nevertheless we consider only one solution branch; hence we presume local uniqueness.

The outputs may be related to energies or forces, stresses or strains, flow-rates or pressure drops, temperatures or fluxes, and are functions of an "input" parameter $P$-vector $\boldsymbol{\mu} \in \mathcal{D} \subset \mathbb{R}^{P}$, which is related to geometry, physical properties, boundary conditions, or loads.

The $\boldsymbol{\mu}$-parametrized nonlinear partial differential equation is written in its variational formulation. The field variable $\boldsymbol{u}^{\mathrm{e}}(\boldsymbol{\mu}) \in X^{\mathrm{e}}$ - say velocity, pressure, or temperature - is then the solution of

$$
a\left(\boldsymbol{u}^{\mathrm{e}}(\boldsymbol{\mu}), \boldsymbol{v} ; \boldsymbol{\mu}\right)=f(\boldsymbol{v} ; \boldsymbol{\mu}), \quad \forall \boldsymbol{v} \in X^{\mathrm{e}}
$$

Here $X^{\mathrm{e}}$ is the appropriate function space with norm $\|\cdot\|_{X}, \boldsymbol{\mu} \equiv\left(\mu_{1} \cdots \mu_{P}\right)$ is in $\mathcal{D} \subset \mathbb{R}^{P}$, and $a(\cdot, \cdot ; \boldsymbol{\mu}): X^{\mathrm{e}} \times X^{\mathrm{e}} \rightarrow \mathbb{R}$ is a parameter-dependent form, which is quadratic with respect to its first argument and linear with respect to the second one. The form $f(\cdot ; \boldsymbol{\mu}): X^{\mathrm{e}} \rightarrow \mathbb{R}$ is parameter-dependent and linear.

We take a "reference" finite element approximation to the exact output and field variable, $s(\boldsymbol{\mu}) \equiv s^{\mathcal{N}}(\boldsymbol{\mu})$ and $\boldsymbol{u}(\boldsymbol{\mu}) \equiv \boldsymbol{u}^{\mathcal{N}}(\boldsymbol{\mu}) \in X^{\mathcal{N}} \equiv X$, for a given $\boldsymbol{\mu} \in \mathcal{D}$, is

$$
\begin{gathered}
s(\boldsymbol{\mu})=\ell(\boldsymbol{u}(\boldsymbol{\mu})) \quad \text { and } \\
a(\boldsymbol{u}(\boldsymbol{\mu}), \boldsymbol{v} ; \boldsymbol{\mu})=f(\boldsymbol{v} ; \boldsymbol{\mu}), \quad \forall \boldsymbol{v} \in X .
\end{gathered}
$$

$\mathcal{N}$ accounts for the number of degrees of freedom in the finite element problem; we assume that $\mathcal{N}$ is chosen sufficiently large so that $s(\boldsymbol{\mu})$ and $\boldsymbol{u}(\boldsymbol{\mu})$ are essentially indistinguishable from $s^{\mathrm{e}}(\boldsymbol{\mu})$ and $\boldsymbol{u}^{\mathrm{e}}(\boldsymbol{\mu})$, respectively.

In many cases, to achieve the desired accuracy, the evaluation $\boldsymbol{\mu} \rightarrow s(\boldsymbol{\mu})$ by discrete projection methods, like finite element or spectral methods, is simply 


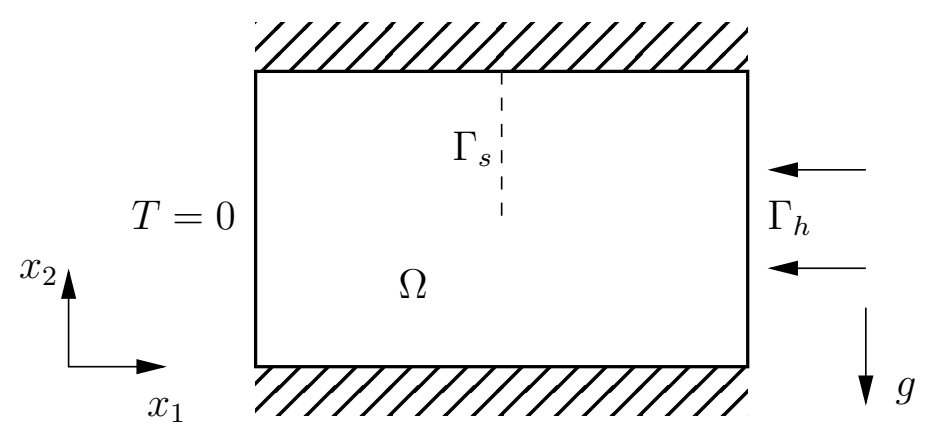

Fig. 1. A closed cavity. On the left the temperature is constant, on the right the heat flux is constant, and the top and the bottom of the cavity are insulated.

too costly, like in many-query or real-time contexts, often of growing interest in engineering. We shall build our reduced basis approximation upon this reference finite element approximation; and we shall evaluate the error in our reduced basis approximation with respect to this reference approximation. The online complexity (and stability) of our reduced basis approach is independent of $\mathcal{N}[20,36,35]$; hence, we may choose $\mathcal{N}$ to be "arbitrarily" large at no detriment to (online) performance. However, in our application, we selected $\mathcal{N}$ as small as possible to provide good accuracy and low computational costs.

\section{A parametrized natural convection problem}

\subsection{Governing equations}

Our natural convection model problem is made up by a two-dimensional rectangular cavity under vertical gravity (Figure 1), with the imposition of a constant temperature on one side, a heated wall on the opposite side, and insulated walls at the top and the bottom of the cavity. We consider three parameters $\boldsymbol{\mu}=\left(\frac{1}{\sqrt{\mathrm{Gr}}}, \frac{1}{\operatorname{Pr} \sqrt{\mathrm{Gr}}}, A\right)$, where Gr is the Grashof number (buoyancy over viscosity ratio), which is defined as $\left(\beta \Delta T g L^{3}\right) / \nu^{2}$ ( $g$ is the gravity constant, $\beta$ is the thermal expansion coefficient, $\Delta T$ is the temperature difference, $L$ is the length scale, and $\nu$ is the kinematic viscosity), $\operatorname{Pr}$ is the Prandtl number (dissipation to conduction ratio), and $A$ is the aspect ratio (actually the length of the cavity). Our field variable $\boldsymbol{u}(\boldsymbol{\mu})=\left(u_{1}, u_{2}, \theta, p, \lambda\right)$ is composed by the velocity components $\left(u_{1}, u_{2}\right)$, the temperature $\theta$, the pressure $p$, and the Lagrange multiplier $\lambda$ related to the zero mean value of the pressure.

For simplicity in the exposition, in this work we identify three test cases, where in turn one of the parameter is fixed. We denote by $\mathcal{D} \subset \mathbb{R}^{3}$ the set of possible/allowed instances of $\boldsymbol{\mu}$. Here $\mathcal{D}$ is actually a hyper-plane of $\mathbb{R}^{3}$ (i.e., only two parameters may vary simultaneously), in a future work we 
will provide an example where $\mathcal{D}$ is truly three-dimensional (i.e., the three parameters may vary simultaneously).

We impose homogeneous Dirichlet boundary conditions for the velocity $\left(u_{1}, u_{2}\right)$, while for the temperature $\theta$ we impose homogeneous Dirichlet boundary conditions on the left side, homogeneous Neumann boundary conditions on the top and the bottom of the cavity, and constant unitary Neumann boundary conditions on the heated side $\Gamma_{h}\left(\partial_{n} \theta=1\right.$ on $\left.\Gamma_{h}\right)$. We scale the pressure so that $\int_{\Omega} p=0$.

We are going to describe our variables with respect to a reference unitary square domain $\Omega$, hence our weak form needs to incorporate a deformation in the $x_{1}$ direction.

The system of partial differential equations in the real rectangular domain $\bar{\Omega}$ reads (with partial derivatives with respect to $\bar{x}_{1}$ and $\bar{x}_{2}$ )

$$
\begin{aligned}
& \left(\left(\frac{\bar{u}_{1}}{\bar{u}_{2}}\right) \cdot \nabla\right)\left(\frac{\bar{u}_{1}}{\bar{u}_{2}}\right)=-\nabla \bar{p}+\frac{1}{\sqrt{\mathrm{Gr}}} \Delta\left(\frac{\bar{u}_{1}}{u_{2}}\right)+\left(\frac{0}{\theta}\right), \\
& \operatorname{div}\left(\frac{\bar{u}_{1}}{\bar{u}_{2}}\right)=0 \\
& \left(\left(\bar{u}_{1}\right) \cdot \nabla\right) \bar{\theta}=\frac{1}{\operatorname{Pr} \sqrt{\mathrm{Gr}}} \Delta \bar{\theta} .
\end{aligned}
$$

We have written the variables in the "real" domain (which is equal to $\Omega$ only for $A=1$ ) with an overline to distinguish from those in the reference domain $\Omega$ that we will use all along this work.

Our outputs of interest are the inverse of the Nusselt number [20] and the flux through the middle half section $\Gamma_{s}$ parallel to $\Gamma_{h}$,

$s_{(1)}(\boldsymbol{\mu})=\frac{1}{\left|\bar{\Gamma}_{h}\right|} \int_{\bar{\Gamma}_{h}} \bar{\theta}(\boldsymbol{\mu})=\frac{1}{\left|\Gamma_{h}\right|} \int_{\Gamma_{h}} \theta(\boldsymbol{\mu}), \quad s_{(2)}(\boldsymbol{\mu})=\frac{1}{\left|\bar{\Gamma}_{s}\right|} \int_{\bar{\Gamma}_{s}} \bar{u}_{1}(\boldsymbol{\mu})=\frac{1}{\left|\Gamma_{s}\right|} \int_{\Gamma_{s}} u_{1}(\boldsymbol{\mu})$.

\subsection{Weak formulation and affine decomposition}

The functional space suited for the weak formulation of our partial differential equation is $X^{\mathrm{e}}=H_{0}^{1}(\Omega) \times H_{0}^{1}(\Omega) \times H^{1}(\Omega) \times L^{2}(\Omega) \times \mathbb{R}$. As introduced in Section 1.2, we use the finite element method. We presume that the number of degrees of freedom $\mathcal{N}$ is so large, that the reference solution $\boldsymbol{u}(\boldsymbol{\mu}) \in X$ and the reference output $l(\boldsymbol{u}(\boldsymbol{\mu}))$ are "indistinguishable" from $\boldsymbol{u}^{\mathrm{e}}(\boldsymbol{\mu}) \in X^{\mathrm{e}}$ and $l\left(\boldsymbol{u}^{\mathrm{e}}(\boldsymbol{\mu})\right)$. We are adopting a $\mathbb{P}^{2}-\mathbb{P}^{2}-\mathbb{P}^{1}$ finite element approximation for velocity, temperature and pressure, respectively.

We take advantage of the affine dependence on the parameters to write the 
weak formulation (2) into its affine components. We denote elements in $X$ as $\boldsymbol{u}=\left(u_{1}, u_{2}, \theta, p, \lambda\right), \boldsymbol{v}=\left(v_{1}, v_{2}, \chi, q, \gamma\right)$, and $\boldsymbol{z}=\left(z_{1}, z_{2}, \zeta, \cdots\right)$. Note that $\lambda(\gamma$ respectively) is a Lagrange multiplier associated with the zero-mean condition on $p$ ( $q$ respectively). The form $a$ can be written as an affine combination of $Q_{0}$ bilinear forms $a_{0}^{q}$ and $Q_{1}$ trilinear forms $a_{1}^{q}$ :

$a(\boldsymbol{u}, \boldsymbol{v} ; \boldsymbol{\mu})=\sum_{q=1}^{Q_{0}} \Theta_{0}^{q}(\boldsymbol{\mu}) a_{0}^{q}(\boldsymbol{u}, \boldsymbol{v})+\frac{1}{2} \sum_{q=1}^{Q_{1}} \Theta_{1}^{q}(\boldsymbol{\mu}) a_{1}^{q}(\boldsymbol{u}, \boldsymbol{u}, \boldsymbol{v}), \quad \forall \boldsymbol{u}, \boldsymbol{v} \in X, \forall \boldsymbol{\mu} \in \mathcal{D}$,

where $\Theta_{0}^{q}, \Theta_{1}^{q}: \mathcal{D} \rightarrow \mathbb{R}$ are parameter-dependent functions and $a_{0}^{q}: X \times X \rightarrow$ $\mathbb{R}, 1 \leq q \leq Q_{0}$, and $a_{1}^{q}: X \times X \times X \rightarrow \mathbb{R}, 1 \leq q \leq Q_{1}$, are parameterindependent continuous bilinear and trilinear forms. (In particular, this assumption presume a quadratic nonlinearity in our partial differential equation.) Without loss of generality, we assume that, for $1 \leq q \leq Q_{1}$ and $\forall \boldsymbol{u}, \boldsymbol{v}, \boldsymbol{w} \in X, a_{1}^{q}(\boldsymbol{u}, \boldsymbol{w}, \boldsymbol{v})=a_{1}^{q}(\boldsymbol{w}, \boldsymbol{u}, \boldsymbol{v})$. We shall further assume that $\Theta_{i}^{q} \in$ $\mathcal{C}^{1}(\mathcal{D}), 1 \leq q \leq Q_{i}, i=0,1$

Three test cases are considered in the following subsections (as a general remark we understand the summation over repeated indices and partial derivatives with respect to $x_{1}$ and $x_{2}$ ). We choose to present three different combinations of the parameters range. In each case we fix one of the parameters and we define the set $\mathcal{D}$ of possible/allowed instances, as well as the corresponding affine decomposition.

\subsection{1 $G r-P r$}

We fix the aspect ratio to 1 and set

$\mathcal{D}=\left\{\boldsymbol{\mu}=\left(\frac{1}{\sqrt{\mathrm{Gr}}}, \frac{1}{\operatorname{Pr} \sqrt{\mathrm{Gr}}}, A\right)\right.$ s.t. $\operatorname{Gr} \in\left[10^{3}, 10^{5}\right], \operatorname{Pr} \in[0.7,7]$, and $\left.A=1\right\}$.

In this case $Q_{0}=3, Q_{1}=1$, and the affine components are:

$$
\begin{array}{llrl}
a_{0}^{1}(\boldsymbol{u}, \boldsymbol{v}) & =-\int_{\Omega} p \partial_{i} v_{i}-\int_{\Omega} q \partial_{i} u_{i}+\lambda \int_{\Omega} p+\gamma \int_{\Omega} q-\int_{\Omega} \theta v_{2} & \Theta_{0}^{1}(\boldsymbol{\mu})=1 & \\
a_{0}^{2}(\boldsymbol{u}, \boldsymbol{v}) & =\int_{\Omega} \partial_{i} u_{j} \partial_{i} v_{j} & \Theta_{0}^{2}(\boldsymbol{\mu})=\mu_{1} & {\left[=\frac{1}{\sqrt{\mathrm{Gr}}}\right]} \\
a_{0}^{3}(\boldsymbol{u}, \boldsymbol{v}) & =\int_{\Omega} \partial_{i} \theta \partial_{i} \chi & \Theta_{0}^{8}(\boldsymbol{\mu})=\mu_{2}\left[=\frac{1}{\operatorname{Pr} \sqrt{\mathrm{Gr}}}\right]
\end{array}
$$

and

$$
a_{1}^{1}(\boldsymbol{u}, \boldsymbol{z}, \boldsymbol{v})=\int_{\Omega} u_{j} \partial_{i} z_{i} v_{j}+\int_{\Omega} z_{j} \partial_{i} u_{i} v_{j}+\int_{\Omega} z_{i} \partial_{i} \theta \chi+\int_{\Omega} u_{i} \partial_{i} \zeta \chi \quad \Theta_{1}^{1}(\boldsymbol{\mu})=1 .
$$




\subsection{2 $G r-A$ :}

We fix the Prandtl number to 7 and set

$\mathcal{D}=\left\{\boldsymbol{\mu}=\left(\frac{1}{\sqrt{\mathrm{Gr}}}, \frac{1}{\operatorname{Pr} \sqrt{\mathrm{Gr}}}, A\right)\right.$ s.t. $\operatorname{Gr} \in\left[5 \cdot 10^{4}, 7.5 \cdot 10^{4}\right], \operatorname{Pr}=7$, and $\left.A \in[1.25,1.5]\right\}$.

Here $Q_{0}=4, Q_{1}=2$, and the affine components are

$$
\begin{array}{llrl}
a_{0}^{1}(\boldsymbol{u}, \boldsymbol{v}) & =-\int_{\Omega} p \partial_{1} v_{1}-\int_{\Omega} q \partial_{1} u_{1} & \Theta_{0}^{1}(\boldsymbol{\mu})=1 & \\
a_{0}^{2}(\boldsymbol{u}, \boldsymbol{v}) & =-\int_{\Omega} p \partial_{2} v_{2}-\int_{\Omega} q \partial_{2} u_{2}-\int_{\Omega} \theta v_{2}+\lambda \int_{\Omega} p+\gamma \int_{\Omega} q & \Theta_{0}^{2}(\boldsymbol{\mu})=\mu_{3} & {[=A]} \\
a_{0}^{3}(\boldsymbol{u}, \boldsymbol{v}) & =\int_{\Omega} \partial_{1} u_{j} \partial_{1} v_{j}+\frac{1}{\operatorname{Pr}} \int_{\Omega} \partial_{1} \theta \partial_{1} \chi & \Theta_{0}^{3}(\boldsymbol{\mu})=\frac{\mu_{1}}{\mu_{3}} \quad\left[=\frac{1}{A \sqrt{\mathrm{Gr}}}\right] \\
a_{0}^{4}(\boldsymbol{u}, \boldsymbol{v})=\int_{\Omega} \partial_{2} u_{j} \partial_{2} v_{j}+\frac{1}{\operatorname{Pr}} \int_{\Omega} \partial_{2} \theta \partial_{2} \chi & \Theta_{0}^{4}(\boldsymbol{\mu})=\mu_{1} \mu_{3} \quad\left[=\frac{A}{\sqrt{\mathrm{Gr}}}\right]
\end{array}
$$

and

$$
\begin{array}{ll}
a_{1}^{1}(\boldsymbol{u}, \boldsymbol{z}, \boldsymbol{v})=\int_{\Omega} u_{j} \partial_{1} z_{1} v_{j}+\int_{\Omega} z_{j} \partial_{1} u_{1} v_{j}+\int_{\Omega} z_{1} \partial_{1} \theta \chi+\int_{\Omega} u_{1} \partial_{1} \zeta \chi & \Theta_{1}^{1}(\boldsymbol{\mu})=1 \\
a_{1}^{2}(\boldsymbol{u}, \boldsymbol{z}, \boldsymbol{v})=\int_{\Omega} u_{j} \partial_{2} z_{2} v_{j}+\int_{\Omega} z_{j} \partial_{2} u_{2} v_{j}+\int_{\Omega} z_{2} \partial_{2} \theta \chi+\int_{\Omega} u_{2} \partial_{2} \zeta \chi & \Theta_{1}^{2}(\boldsymbol{\mu})=\mu_{3}[=A] .
\end{array}
$$

\subsection{3 $\operatorname{Pr}-A$}

We fix the Grashof number to $10^{5}$ and set

$\mathcal{D}=\left\{\boldsymbol{\mu}=\left(\frac{1}{\sqrt{\mathrm{Gr}}}, \frac{1}{\operatorname{Pr} \sqrt{\mathrm{Gr}}}, A\right)\right.$ s.t. $\mathrm{Gr}=10^{5}, \operatorname{Pr} \in[0.7,7]$, and $\left.A \in[1.2,1.4]\right\}$.

Then $Q_{0}=5, Q_{1}=2$, and the affine components are

$$
\begin{array}{rlrl}
a_{0}^{1}(\boldsymbol{u}, \boldsymbol{v})= & -\int_{\Omega} p \partial_{1} v_{1}-\int_{\Omega} q \partial_{1} u_{1} & \Theta_{0}^{1}(\boldsymbol{\mu})=1 & \\
a_{0}^{2}(\boldsymbol{u}, \boldsymbol{v})= & -\int_{\Omega} p \partial_{2} v_{2}-\int_{\Omega} q \partial_{2} u_{2}-\int_{\Omega} \theta v_{2} & & \\
& +\frac{1}{\sqrt{\mathrm{Gr}}} \int_{\Omega} \partial_{2} u_{j} \partial_{2} v_{j}+\lambda \int_{\Omega} p+\gamma \int_{\Omega} q & \Theta_{0}^{2}(\boldsymbol{\mu})=\mu_{3} & {[=A]} \\
a_{0}^{3}(\boldsymbol{u}, \boldsymbol{v})= & \frac{1}{\sqrt{\mathrm{Gr}}} \int_{\Omega} \partial_{1} u_{j} \partial_{1} v_{j} & \Theta_{0}^{3}(\boldsymbol{\mu})=\frac{1}{\mu_{3}} & {\left[=\frac{1}{A}\right]} \\
a_{0}^{4}(\boldsymbol{u}, \boldsymbol{v})= & \int_{\Omega} \partial_{1} \theta \partial_{1} \chi & \Theta_{0}^{4}(\boldsymbol{\mu})=\frac{\mu_{2}}{\mu_{3}}\left[=\frac{1}{A \operatorname{Pr} \sqrt{\mathrm{Gr}}}\right] \\
a_{0}^{5}(\boldsymbol{u}, \boldsymbol{v})= & \int_{\Omega} \partial_{2} \theta \partial_{2} \chi & \Theta_{0}^{5}(\boldsymbol{\mu})=\mu_{2} \mu_{3}\left[=\frac{A}{\operatorname{Pr} \sqrt{\mathrm{Gr}}}\right]
\end{array}
$$


and

$$
\begin{array}{ll}
a_{1}^{1}(\boldsymbol{u}, \boldsymbol{z}, \boldsymbol{v})=\int_{\Omega} u_{j} \partial_{1} z_{1} v_{j}+\int_{\Omega} z_{j} \partial_{1} u_{1} v_{j}+\int_{\Omega} z_{1} \partial_{1} \theta \chi+\int_{\Omega} u_{1} \partial_{1} \zeta \chi & \Theta_{1}^{1}(\boldsymbol{\mu})=1 \\
a_{1}^{2}(\boldsymbol{u}, \boldsymbol{z}, \boldsymbol{v})=\int_{\Omega} u_{j} \partial_{2} z_{2} v_{j}+\int_{\Omega} z_{j} \partial_{2} u_{2} v_{j}+\int_{\Omega} z_{2} \partial_{2} \theta \chi+\int_{\Omega} u_{2} \partial_{2} \zeta \chi & \Theta_{1}^{2}(\boldsymbol{\mu})=\mu_{3}[=A] .
\end{array}
$$

In the three cases, the right hand side, which linearly depends on $\mu_{1}$, and the (linear) outputs take the form

$$
\begin{aligned}
f(\boldsymbol{v} ; \boldsymbol{\mu}) & =\mu_{1} \int_{\Gamma_{h}} \chi \\
\ell_{(1)}(\boldsymbol{v}) & =\int_{\Gamma_{h}} \chi \\
\ell_{(2)}(\boldsymbol{v}) & =\int_{\Gamma_{s}} v_{1} .
\end{aligned}
$$

\subsection{Reduced basis formulation and natural norms}

In the offline stage we build a reduced space $W_{N}$ generated by a set of finite element solutions $\boldsymbol{u}\left(\boldsymbol{\mu}^{(n)}\right), n=1, \ldots, N$ for properly selected parameters $\boldsymbol{\mu}^{(n)}$, $n=1, \ldots, N$ (snapshots). (In [30] a greedy selection algorithm is described in details.) In the online stage, given a parameter instance $\boldsymbol{\mu}$, we have to solve the problem: find $\boldsymbol{u}_{N}(\boldsymbol{\mu})$ in $W_{N}$, such that

$$
a\left(\boldsymbol{u}_{N}(\boldsymbol{\mu}), \boldsymbol{v} ; \boldsymbol{\mu}\right)=f(\boldsymbol{v} ; \boldsymbol{\mu}), \quad \forall \boldsymbol{v} \in W_{N} .
$$

Here $W_{N}$ is a problem-specific space of dimension related to $N \leq N_{\max }$ (for each physical component: velocity, pressure, temperature) that focuses on the (typically very smooth) parametric manifold of interest $-\left\{\boldsymbol{u}^{\mathrm{e}}(\boldsymbol{\mu}) \mid \boldsymbol{\mu} \in \mathcal{D}\right\}$ - and thus the reduced basis method enjoys very rapid convergence as $N$ increases $[5,16] ; N_{\max }$ is related to the maximal size of our reduced basis.

We are going to compute sharp and rigorous error bound $\Delta_{N}(\boldsymbol{\mu})$ over the solution with respect to a norm $\||\cdot|\|$, to be defined in the following, and an error bound $\Delta_{N}^{s}(\boldsymbol{\mu})$ on the output, such that

$$
\begin{gathered}
\left\|\boldsymbol{u}^{\mathcal{N}}(\boldsymbol{\mu})-\boldsymbol{u}_{N}(\boldsymbol{\mu}) \mid\right\| \leq \Delta_{N}(\boldsymbol{\mu}) \quad \text { and } \\
\left|s^{\mathcal{N}}(\boldsymbol{\mu})-s_{N}(\boldsymbol{\mu})\right| \leq \Delta_{N}^{s}(\boldsymbol{\mu}), \quad \forall \boldsymbol{\mu} \in \mathcal{D},
\end{gathered}
$$

so that we can efficiently determine if $N$ is too small - and our reduced basis approximation unacceptably inaccurate — or if $N$ is too large - and our reduced basis approximation unnecessarily expensive. Without rigorous 
and fast error bounds we can not determine in "real-time" if critical design conditions and constraints are satisfied.

At present, there are only preliminary results on a priori error estimates in the reduced basis method $[16,17,21]$ for linear problems. Instead we apply the a posteriori analysis as carried out in [3] which is based on the natural norm approach, first introduced for the linear case in [35]. This relies on the following definitions.

We denote the inner product and norm associated with our Hilbert space $X\left(\equiv X^{\mathcal{N}}\right.$, finite dimensional $)$ as $(\boldsymbol{w}, \boldsymbol{v})_{X}$ and $\|\boldsymbol{v}\|_{X}=\sqrt{(\boldsymbol{v}, \boldsymbol{v})_{X}}$, respectively. We further define the dual norm for any bounded linear functional $f$ as

$$
\|f\|_{X^{\prime}} \equiv \sup _{\boldsymbol{v} \in X} \frac{f(\boldsymbol{v})}{\|\boldsymbol{v}\|_{X}}
$$

Recalling (4) and the symmetry of the $a_{1}^{q}$ 's in the first two variables, the Fréchet derivative of $a(\cdot, \cdot ; \boldsymbol{\mu})$ with respect to the first variable at a point $\boldsymbol{w} \in X$ can be expressed as

$d a(\boldsymbol{w} ; \boldsymbol{\mu})(\boldsymbol{u}, \boldsymbol{v})=\sum_{q=1}^{Q_{0}} \Theta_{0}^{q}(\boldsymbol{\mu}) a_{0}^{q}(\boldsymbol{u}, \boldsymbol{v})+\sum_{q=1}^{Q_{1}} \Theta_{1}^{q}(\boldsymbol{\mu}) a_{1}^{q}(\boldsymbol{u}, \boldsymbol{w}, \boldsymbol{v}), \quad \forall \boldsymbol{u}, \boldsymbol{v} \in X, \forall \boldsymbol{\mu} \in \mathcal{D}$.

For any $\boldsymbol{\mu}$ in $\mathcal{D}$ and any solution $\boldsymbol{u}(\boldsymbol{\mu})$ in the region of our interest, we assume that $d a(\boldsymbol{u}(\boldsymbol{\mu}) ; \boldsymbol{\mu})$ is "stable" and continuous in the sense that there exist $\beta_{0}>0$ and $\gamma_{0} \in \mathbb{R}$ such that $\forall \boldsymbol{\mu} \in \mathcal{D}$,

$$
\begin{aligned}
& 0<\beta_{0}<\beta(\boldsymbol{\mu}) \equiv \inf _{\boldsymbol{w} \in X} \sup _{\boldsymbol{v} \in X} \frac{d a(\boldsymbol{u}(\boldsymbol{\mu}) ; \boldsymbol{\mu})(\boldsymbol{w}, \boldsymbol{v})}{\|\boldsymbol{w}\|_{X}\|\boldsymbol{v}\|_{X}}, \\
& \infty>\gamma_{0}>\gamma(\boldsymbol{\mu}) \equiv \sup _{\boldsymbol{w} \in X} \sup _{\boldsymbol{v} \in X} \frac{d a(\boldsymbol{u}(\boldsymbol{\mu}) ; \boldsymbol{\mu})(\boldsymbol{w}, \boldsymbol{v})}{\|\boldsymbol{w}\|_{X}\|\boldsymbol{v}\|_{X}} .
\end{aligned}
$$

It then follows that in the neighborhood of $\boldsymbol{u}(\boldsymbol{\mu})$ the solution is unique. We further assume that $\ell$ and $f$ are in $X^{\prime}$, i.e., they are bounded linear functionals.

Let $\boldsymbol{u}_{N}(\boldsymbol{\mu})$ be the reduced basis approximation to $\boldsymbol{u}(\boldsymbol{\mu})$ given by (8). We next introduce $[38,20,9,15,35]$ the parametrized linear operator $T_{N}^{\mu}: X \rightarrow X$, such that

$$
\left(T_{N}^{\boldsymbol{\mu}} \boldsymbol{w}, \boldsymbol{v}\right)_{X}=d a\left(\boldsymbol{u}_{N}(\boldsymbol{\mu}) ; \boldsymbol{\mu}\right)(\boldsymbol{w}, \boldsymbol{v}), \forall \boldsymbol{v}, \boldsymbol{w} \in X
$$

Our method, in particular the inf-sup constant lower bound construction, requires a discrete set of $K$ parameter values, $\mathcal{V}^{K} \equiv\left\{\overline{\boldsymbol{\mu}}_{1}, \ldots, \overline{\boldsymbol{\mu}}_{K}\right\} \subset \mathcal{D}$, upon which to construct local norms; a fixed integer $\bar{N} \leq N_{\max }$; and an indicator 
function $\mathcal{I}^{K}: \mathcal{D} \rightarrow \mathcal{V}^{K}$ which associates to any $\boldsymbol{\mu}$ in $\mathcal{D}$ a member of $\mathcal{V}^{K}$. (The process by which we select "good" $\mathcal{V}^{K}$ and $\mathcal{I}^{K}$ is briefly described in Section 3.)

We assume that, $\forall \overline{\boldsymbol{\mu}} \in \mathcal{V}^{K}$,

$$
\begin{aligned}
& 0<\beta_{\bar{N}}(\overline{\boldsymbol{\mu}}) \equiv \inf _{\boldsymbol{w} \in X} \sup _{\boldsymbol{v} \in X} \frac{d a\left(\boldsymbol{u}_{\bar{N}}(\overline{\boldsymbol{\mu}}) ; \overline{\boldsymbol{\mu}}\right)(\boldsymbol{w}, \boldsymbol{v})}{\|\boldsymbol{w}\|_{X}\|\boldsymbol{v}\|_{X}} \equiv \inf _{\boldsymbol{w} \in X} \frac{\left\|T_{\overline{\boldsymbol{\mu}}}^{\overline{\boldsymbol{w}}} \boldsymbol{w}\right\|_{X}}{\|\boldsymbol{w}\|_{X}}, \\
& \quad \infty>\gamma_{\bar{N}}(\overline{\boldsymbol{\mu}}) \equiv \sup _{\boldsymbol{w} \in X} \sup _{\boldsymbol{v} \in X} \frac{d a\left(\boldsymbol{u}_{\bar{N}}(\overline{\boldsymbol{\mu}}) ; \overline{\boldsymbol{\mu}}\right)(\boldsymbol{w}, \boldsymbol{v})}{\|\boldsymbol{w}\|_{X}\|\boldsymbol{v}\|_{X}} \equiv \sup _{\boldsymbol{w} \in X} \frac{\left\|T_{\bar{\mu}}^{\bar{\mu}} \boldsymbol{w}\right\|_{X}}{\|\boldsymbol{w}\|_{X}} .
\end{aligned}
$$

Note that this is true if $\boldsymbol{u}_{\bar{N}}(\overline{\boldsymbol{\mu}})$ is close enough to $\boldsymbol{u}(\overline{\boldsymbol{\mu}})$ (even though this is not required here); from the Cauchy-Schwarz inequality, $\boldsymbol{v}=T_{\bar{N}}^{\bar{\mu}} \boldsymbol{w}$ is the inner supremizer in (11) and (12).

We then introduce the "natural norm"

$$
\|\boldsymbol{v}\|_{\overline{\boldsymbol{\mu}}} \equiv\left\|T_{\bar{\mu}}^{\bar{\mu}} \boldsymbol{v}\right\|_{X}, \quad \forall \boldsymbol{v} \in X
$$

(To simplify the notation we have dropped the index ${ }_{N}$ from the norm symbol.) This norm is the extension of the natural norm introduced in [35] for the linear case and it was first introduced in [3]. Note that, thanks to our assumptions (11) and (12) on $\beta_{\bar{N}}(\overline{\boldsymbol{\mu}})$ and $\gamma_{\bar{N}}(\overline{\boldsymbol{\mu}}),(13)$ does indeed define a norm, which is equivalent to $\|\cdot\|_{X}$.

\subsection{Trilinear forms continuity constants}

In the development of the error bound, in [3] it is required that there exists a positive $\rho_{\overline{\boldsymbol{\mu}}}(\boldsymbol{\mu})$ such that

$$
\left|d a\left(\boldsymbol{z}^{2} ; \boldsymbol{\mu}\right)(\boldsymbol{v}, \boldsymbol{w})-d a\left(\boldsymbol{z}^{1} ; \boldsymbol{\mu}\right)(\boldsymbol{v}, \boldsymbol{w})\right| \leq \rho_{\overline{\boldsymbol{\mu}}}(\boldsymbol{\mu})\left\|\left|\boldsymbol{z}^{2}-\boldsymbol{z}^{1}\right|\right\|_{\overline{\boldsymbol{\mu}}}\|\boldsymbol{v}\|\left\|_{\overline{\boldsymbol{\mu}}}\right\| \boldsymbol{w} \|_{X}
$$

for all $\boldsymbol{z}^{1}, \boldsymbol{z}^{2}, \boldsymbol{v}$, and $\boldsymbol{w}$ in $X$ and similarly, in the construction of an inf-sup lower bound, that there exists a positive $\rho_{X, \overline{\boldsymbol{\mu}}}$ such that

$$
\begin{gathered}
\left|\sum_{q=1}^{Q_{1}} \Theta_{1}^{q}(\overline{\boldsymbol{\mu}}) a_{1}^{q}(\boldsymbol{v}, \boldsymbol{z}, \boldsymbol{w})\right| \leq \rho_{X, \overline{\boldsymbol{\mu}}}\|\boldsymbol{z}\|_{X}\|\boldsymbol{v}\|\left\|_{\overline{\boldsymbol{\mu}}}\right\| \boldsymbol{w} \|_{X}, \\
\left|\sum_{q=1}^{Q_{1}}\left(\Theta_{1}^{q}(\boldsymbol{\mu})-\Theta_{1}^{q}(\overline{\boldsymbol{\mu}})\right) a_{1}^{q}(\boldsymbol{v}, \boldsymbol{z}, \boldsymbol{w})\right| \leq \max _{q=1, \ldots, Q_{1}} \frac{\left|\Theta_{1}^{q}(\boldsymbol{\mu})-\Theta_{1}^{q}(\overline{\boldsymbol{\mu}})\right|}{\left|\Theta_{1}^{q}(\overline{\boldsymbol{\mu}})\right|} \rho_{X, \overline{\boldsymbol{\mu}}}\|\boldsymbol{z}\|_{X}\|\mid \boldsymbol{v}\|\left\|_{\overline{\boldsymbol{\mu}}}\right\| \boldsymbol{w} \|_{X},
\end{gathered}
$$

for all $\boldsymbol{z}, \boldsymbol{v}$, and $\boldsymbol{w}$ in $X$. 
We have to provide these constants; from [3], section 6.3 we know that

$$
\begin{gathered}
\left|a_{1}^{1}(\boldsymbol{v},, \boldsymbol{z}, \boldsymbol{w})+a_{1}^{2}(\boldsymbol{v},, \boldsymbol{z}, \boldsymbol{w})\right| \leq\left(\frac{\rho_{\overline{\boldsymbol{\mu}}} \rho_{X}}{\beta(\overline{\boldsymbol{\mu}})}+2 \rho_{\overline{\boldsymbol{\mu}}}^{2}\right)\|\boldsymbol{z}\|\left\|_{\overline{\boldsymbol{\mu}}}\right\| \boldsymbol{v}\|\|_{\overline{\boldsymbol{\mu}}}\|\boldsymbol{w}\|_{X} \text {, and } \\
\left|a_{1}^{1}(\boldsymbol{v}, \boldsymbol{z}, \boldsymbol{w})+a_{1}^{2}(\boldsymbol{v},, \boldsymbol{z}, \boldsymbol{w})\right| \leq 3 \rho_{\overline{\boldsymbol{\mu}}} \rho_{X}\|\boldsymbol{z}\|_{X}\|\boldsymbol{v}\|_{\overline{\boldsymbol{\mu}}}\|\boldsymbol{w}\|_{X},
\end{gathered}
$$

where the constants $\rho_{X}$ and $\rho_{\overline{\boldsymbol{\mu}}}$ are such that (cf. the Sobolev embedding theorem)

$$
|\boldsymbol{v}|_{4} \leq \rho_{X}\|\boldsymbol{v}\|_{X} \text { and }|\boldsymbol{v}|_{4} \leq \rho_{\overline{\boldsymbol{\mu}}}\|\boldsymbol{v}\|_{\overline{\boldsymbol{\mu}}} \text { for all } \boldsymbol{v} \in X
$$

and the semi-norm $|\cdot|_{4}$ as the restriction of the $L^{4}$-norm to the velocity and temperature fields,

$$
|\boldsymbol{v}|_{4}^{4}=\int_{\Omega}\left(v_{1}^{2}+v_{2}^{2}+\chi^{2}\right)^{2} .
$$

Then, since $\Theta_{1}^{1}(\boldsymbol{\mu})=1$ and $\Theta_{1}^{2}(\boldsymbol{\mu})=\mu_{3}=A$, by taking

$$
\rho_{\overline{\boldsymbol{\mu}}}(\boldsymbol{\mu}) \equiv\left(\frac{\rho_{\overline{\boldsymbol{\mu}}} \rho_{X}}{\beta(\overline{\boldsymbol{\mu}})}+2 \rho_{\overline{\boldsymbol{\mu}}}^{2}\right) \max \left\{1, \mu_{3}\right\} \quad \text { and } \quad \rho_{X, \overline{\boldsymbol{\mu}}} \equiv 3 \rho_{\overline{\boldsymbol{\mu}}} \rho_{X} \max \left\{1, \bar{\mu}_{3}\right\}
$$

inequalities (14), (15), and (16) are satisfied.

\subsection{Existence and uniqueness}

The results in [3], inequalities (15),(16), and definitions (17) allow to state the following existence, uniqueness, and error estimate results. Let $\boldsymbol{\mu} \in \mathcal{D}$, $\overline{\boldsymbol{\mu}} \in \mathcal{V}^{K}, N \leq N_{\max }$, and $\bar{N} \leq N_{\max }$, we define (implicitly dropping some of the subscripts $\bar{N}$ and ${ }_{N}$ )

$$
\begin{aligned}
& \beta_{\overline{\boldsymbol{\mu}}}(\boldsymbol{\mu}) \equiv \inf _{\boldsymbol{w} \in X} \sup _{\boldsymbol{v} \in X} \frac{\left(T_{N}^{\boldsymbol{\mu}} \boldsymbol{w}, \boldsymbol{v}\right)_{X}}{\|\boldsymbol{w}\|_{\overline{\boldsymbol{\mu}}}\|\boldsymbol{v}\|_{X}}=\inf _{\boldsymbol{w} \in X} \frac{\left\|T_{N}^{\boldsymbol{\mu}} \boldsymbol{w}\right\|_{X}}{\|\boldsymbol{w}\|_{\overline{\boldsymbol{\mu}}}}=\inf _{\boldsymbol{w} \in X} \frac{\left\|T_{N}^{\boldsymbol{\mu}} \boldsymbol{w}\right\|_{X}}{\left\|T_{\bar{\mu}}^{\bar{\mu}} \boldsymbol{w}\right\|_{X}}, \\
& \gamma_{\overline{\boldsymbol{\mu}}}(\boldsymbol{\mu}) \equiv \sup _{\boldsymbol{w} \in X} \sup _{\boldsymbol{v} \in X} \frac{\left(T_{N}^{\boldsymbol{\mu}} \boldsymbol{w}, \boldsymbol{v}\right)_{X}}{\|\boldsymbol{w}\|_{\overline{\boldsymbol{\mu}}}\|\boldsymbol{v}\|_{X}}=\sup _{\boldsymbol{w} \in X} \frac{\left\|T_{N}^{\boldsymbol{\mu}} \boldsymbol{w}\right\|_{X}}{\|\boldsymbol{w}\|_{\overline{\boldsymbol{\mu}}}}=\sup _{\boldsymbol{w} \in X} \frac{\left\|T_{N}^{\boldsymbol{\mu}} \boldsymbol{w}\right\|_{X}}{\left\|T_{\overline{\boldsymbol{\mu}}}^{\bar{\mu}} \boldsymbol{w}\right\|_{X}} .
\end{aligned}
$$

In Section 2.6 we recall how to compute an explicit rigorous lower bound $\bar{B}_{\overline{\boldsymbol{\mu}}}^{L B}(\boldsymbol{\mu}) \leq \beta_{\overline{\boldsymbol{\mu}}}(\boldsymbol{\mu})$. We can assume that for at least a $\overline{\boldsymbol{\mu}}$ in $\mathcal{V}^{K}$ the lower bound $\bar{B}_{\overline{\boldsymbol{\mu}}}^{L B}(\boldsymbol{\mu})$ is positive. If this is not possible, then we are not able to give an error estimate for that particular $\boldsymbol{\mu}$. In this case, we would have to enrich $\mathcal{V}^{K}$ and provide a natural norm "near" to this $\boldsymbol{\mu}$. 
We define

$$
\langle A(\boldsymbol{u} ; \boldsymbol{\mu}), \boldsymbol{v}\rangle=a(\boldsymbol{u}, \boldsymbol{v} ; \boldsymbol{\mu})-f(\boldsymbol{v}) \forall \boldsymbol{u}, \boldsymbol{v} \in X
$$

We also introduce the following quantities:

$$
\begin{aligned}
\epsilon_{N}(\boldsymbol{\mu}) & \equiv\left\|A\left(\boldsymbol{u}_{N}(\boldsymbol{\mu}) ; \boldsymbol{\mu}\right)\right\|_{X^{\prime}}, \\
\tau_{N, \overline{\boldsymbol{\mu}}}(\boldsymbol{\mu}) & \equiv \frac{2 \rho_{\overline{\boldsymbol{\mu}}}(\boldsymbol{\mu}) \epsilon_{N}(\boldsymbol{\mu})}{\bar{B}_{\overline{\boldsymbol{\mu}}}^{L B}(\boldsymbol{\mu})^{2}} \\
\Delta_{N, \overline{\boldsymbol{\mu}}}(\boldsymbol{\mu}) & \equiv \frac{\bar{B}_{\overline{\boldsymbol{\mu}}}^{L B}(\boldsymbol{\mu})}{\rho_{\overline{\boldsymbol{\mu}}}(\boldsymbol{\mu})}\left[1-\sqrt{1-\tau_{N, \overline{\boldsymbol{\mu}}}(\boldsymbol{\mu})}\right] .
\end{aligned}
$$

Theorem 1 (Theorem 3.3 in [3]) Let $\boldsymbol{\mu} \in \mathcal{D}$ and assume that for a $\overline{\boldsymbol{\mu}} \in$ $\mathcal{V}^{K}, \bar{B}_{\overline{\boldsymbol{\mu}}}^{L B}(\boldsymbol{\mu})>0$ and $\tau_{\overline{\boldsymbol{\mu}}}(\boldsymbol{\mu}) \leq 1$. Then there exists a solution $\boldsymbol{u}(\boldsymbol{\mu})$ to (3) such that

$$
\left\|\boldsymbol{u}(\boldsymbol{\mu})-\boldsymbol{u}_{N}(\boldsymbol{\mu})\right\|_{\bar{\mu}} \leq \Delta_{N, \bar{\mu}}(\boldsymbol{\mu}) ;
$$

moreover this is the unique one satisfying

$$
\left\|\boldsymbol{u}(\boldsymbol{\mu})-\boldsymbol{u}_{N}(\boldsymbol{\mu})\right\|_{\overline{\boldsymbol{\mu}}}<\frac{\bar{B}_{\overline{\boldsymbol{\mu}}}^{L B}(\boldsymbol{\mu})}{\rho_{\overline{\boldsymbol{\mu}}}(\boldsymbol{\mu})} ;
$$

the effectivity of the error bound (21) is

$$
\Delta_{N, \overline{\boldsymbol{\mu}}}(\boldsymbol{\mu}) \leq\left[\frac{2 \gamma_{\overline{\boldsymbol{\mu}}}(\boldsymbol{\mu})}{\bar{B}_{\overline{\boldsymbol{\mu}}}^{L B}(\boldsymbol{\mu})}+\tau_{\overline{\boldsymbol{\mu}}}(\boldsymbol{\mu})\right]\left\|\boldsymbol{u}(\boldsymbol{\mu})-\boldsymbol{u}_{N}(\boldsymbol{\mu})\right\|_{\overline{\boldsymbol{\mu}}}
$$

As a corollary, to compute an error bound for the output, we need the natural dual norm of the output linear functional,

$$
\|\| \ell \|_{\overline{\boldsymbol{\mu}}^{\prime}}=\sup _{\boldsymbol{v} \in Y} \frac{|\ell(\boldsymbol{v})|}{\|\boldsymbol{v}\|_{\overline{\boldsymbol{\mu}}}} .
$$

If $\bar{B}_{\overline{\boldsymbol{\mu}}}^{L B}(\boldsymbol{\mu})>0$ and $\tau_{N, \overline{\boldsymbol{\mu}}}(\boldsymbol{\mu}) \leq 1$, we can then state the output error bound as

$$
\left|s(\boldsymbol{\mu})-s_{N}(\boldsymbol{\mu})\right| \leq \| \ell||_{\overline{\boldsymbol{\mu}}^{\prime}} \Delta_{N, \overline{\boldsymbol{\mu}}}(\boldsymbol{\mu}) \equiv \Delta_{N, \overline{\boldsymbol{\mu}}}^{s}(\boldsymbol{\mu}) .
$$

We do not have any result on the effectivity of the output error bound. Another possibility, that would lead to a smaller effectivity, is reported in the Appendix of [3], is the use of an adjoint problem. 


\subsection{Lower bound of the inf-sup constant}

We recall the construction of a lower bound $\bar{B}_{\overline{\boldsymbol{\mu}}}^{L B}(\boldsymbol{\mu})$ to the inf-sup parameter $\beta_{\overline{\boldsymbol{\mu}}}(\boldsymbol{\mu})$ proposed in [3], which is an extension of the one proposed in [35] for linear problems.

For $\boldsymbol{\mu} \in \mathcal{D}$ and $\overline{\boldsymbol{\mu}} \in \mathcal{V}^{K}$, let

$$
\boldsymbol{\alpha}(\boldsymbol{\mu}, \overline{\boldsymbol{\mu}})=\boldsymbol{u}_{N}(\boldsymbol{\mu})-\boldsymbol{u}_{\bar{N}}(\overline{\boldsymbol{\mu}})-\sum_{p=1}^{P}\left(\mu_{p}-\bar{\mu}_{p}\right) \frac{\partial \boldsymbol{u}_{\bar{N}}}{\partial \mu_{p}}(\overline{\boldsymbol{\mu}}) \in W_{N_{\max }}
$$

and for $p=1, \ldots, P$ let

$a_{p}^{\overline{\boldsymbol{\mu}}}(\cdot, \cdot) \equiv \sum_{q=1}^{Q_{0}} \frac{\partial \Theta_{0}^{q}}{\partial \mu_{p}}(\overline{\boldsymbol{\mu}}) a_{0}^{q}(\cdot, \cdot)+\sum_{q=1}^{Q_{1}} \frac{\partial \Theta_{1}^{q}}{\partial \mu_{p}}(\overline{\boldsymbol{\mu}}) a_{1}^{q}\left(\cdot, \boldsymbol{u}_{\bar{N}}(\overline{\boldsymbol{\mu}}), \cdot\right)+\sum_{q=1}^{Q_{1}} \Theta_{1}^{q}(\overline{\boldsymbol{\mu}}) a_{1}^{q}\left(\cdot, \frac{\partial \boldsymbol{u}_{\bar{N}}}{\partial \mu_{p}}(\overline{\boldsymbol{\mu}}), \cdot\right)$.

We need to compute the extreme generalized eigenvalues with respect to $\||\cdot|\|_{\bar{\mu}}$ of $a_{p}^{\bar{\mu}}, a_{0}^{q}$ and $a_{1}^{q}\left(\cdot, \boldsymbol{u}_{\bar{N}}(\overline{\boldsymbol{\mu}}), \cdot\right)$ as

$$
\begin{aligned}
& \lambda_{p, \text { inf }}^{\bar{\mu}}=\inf _{\boldsymbol{w} \in X} \frac{a_{p}^{\bar{\mu}}\left(\boldsymbol{w}, T \frac{\bar{\mu}}{N} \boldsymbol{w}\right)}{\|\boldsymbol{w}\|_{\bar{\mu}}^{2}}, \quad \lambda_{p, \text { sup }}^{\overline{\boldsymbol{\mu}}}=\sup _{\boldsymbol{w} \in X} \frac{a_{p}^{\overline{\boldsymbol{\mu}}}\left(\boldsymbol{w}, T \frac{\overline{\boldsymbol{\mu}}}{N} \boldsymbol{w}\right)}{\|\boldsymbol{w}\|_{\bar{\mu}}^{2}}, \quad p=1, \ldots, P \\
& \gamma_{0, q, \text { inf }}^{\overline{\boldsymbol{\mu}}}=\inf _{\boldsymbol{w} \in X} \frac{a_{0}^{q}\left(\boldsymbol{w}, T \frac{\overline{\boldsymbol{\mu}}}{N} \boldsymbol{w}\right)}{\|\boldsymbol{w}\|_{\overline{\boldsymbol{\mu}}}^{2}}, \quad \gamma_{0, q, \text { sup }}^{\overline{\boldsymbol{\mu}}}=\sup _{\boldsymbol{w} \in X} \frac{a_{0}^{q}\left(\boldsymbol{w}, T \frac{\overline{\boldsymbol{\mu}}}{N} \boldsymbol{w}\right)}{\|\boldsymbol{w}\|_{\overline{\boldsymbol{\mu}}}^{2}}, \quad q=1, \ldots, Q_{0}, \\
& \gamma_{1, q, \inf }^{\overline{\boldsymbol{\mu}}}=\inf _{\boldsymbol{w} \in X} \frac{a_{1}^{q}\left(\boldsymbol{w}, \boldsymbol{u}_{\bar{N}}(\overline{\boldsymbol{\mu}}), T_{\bar{N}}^{\overline{\boldsymbol{\mu}}} \boldsymbol{w}\right)}{\|\boldsymbol{w}\|_{\overline{\boldsymbol{\mu}}}^{2}}, \quad \gamma_{1, q, \sup }^{\overline{\boldsymbol{\mu}}}=\sup _{\boldsymbol{w} \in X} \frac{a_{1}^{q}\left(\boldsymbol{w}, \boldsymbol{u}_{\bar{N}}(\overline{\boldsymbol{\mu}}), T_{\bar{N}}^{\overline{\boldsymbol{\mu}} \boldsymbol{w})}\right.}{\|\boldsymbol{w}\|_{\overline{\boldsymbol{\mu}}}^{2}}, \quad q=1, \ldots, Q_{1} .
\end{aligned}
$$

The computation of the extreme eigenvalues $\lambda_{p, \text { inf } \mid \text { sup }}^{\bar{\mu}}, p=1, \ldots, P, \gamma_{i, q, \text { inf } \mid \text { sup }}^{\bar{\mu}}$, $q=1, \ldots, Q_{i}, i=0,1$, is done for all $\overline{\boldsymbol{\mu}}$ in $\mathcal{V}^{K}$.

In [3] it is shown that a rigorous lower bound to $\beta_{\overline{\boldsymbol{\mu}}}(\boldsymbol{\mu})$ is given by

$$
\begin{aligned}
\bar{B}_{\overline{\boldsymbol{\mu}}}^{L B}(\boldsymbol{\mu}) & \equiv 1+\sum_{p=1}^{P} \min _{\lambda_{p}=\lambda_{p, \text { inf } \mid \text { sup }}^{\bar{\mu}}}\left(\mu_{p}-\bar{\mu}_{p}\right) \lambda_{p} \\
& +\sum_{q=1}^{Q_{0}} \min _{\gamma_{q}=\gamma_{0, q, \mathrm{inf} \mid \sup }^{\bar{\mu}}}\left(\Theta_{0}^{q}(\boldsymbol{\mu})-\Theta_{0}^{q}(\overline{\boldsymbol{\mu}})-\sum_{p=1}^{P}\left(\mu_{p}-\bar{\mu}_{p}\right) \frac{\partial \Theta_{0}^{q}}{\partial \mu_{p}}(\overline{\boldsymbol{\mu}})\right) \gamma_{q} \\
& +\sum_{q=1}^{Q_{1}} \min _{\gamma_{q}=\gamma_{1, q, \mathrm{inf} \mid \sup }^{\bar{\mu}}}\left(\Theta_{1}^{q}(\boldsymbol{\mu})-\Theta_{1}^{q}(\overline{\boldsymbol{\mu}})-\sum_{p=1}^{P}\left(\mu_{p}-\bar{\mu}_{p}\right) \frac{\partial \Theta_{1}^{q}}{\partial \mu_{p}}(\overline{\boldsymbol{\mu}})\right) \gamma_{q} \\
& -\rho_{X, \overline{\boldsymbol{\mu}}}\left(\|\boldsymbol{\alpha}(\boldsymbol{\mu}, \overline{\boldsymbol{\mu}})\|_{X}+\max _{q=1, \ldots, Q_{1}} \frac{\left|\Theta_{1}^{q}(\boldsymbol{\mu})-\Theta_{1}^{q}(\overline{\boldsymbol{\mu}})\right|}{\left|\Theta_{1}^{q}(\overline{\boldsymbol{\mu}})\right|}\left\|\boldsymbol{u}_{N}(\boldsymbol{\mu})-\boldsymbol{u}_{\bar{N}}(\overline{\boldsymbol{\mu}})\right\|_{X}\right),
\end{aligned}
$$


where we denote the minimum of a function $g$ over two values as

$$
\min _{\lambda=\lambda_{\text {inf } \mid \text { sup }}} g(\lambda) \equiv \min \left\{g\left(\lambda_{\text {inf }}\right), g\left(\lambda_{\text {sup }}\right)\right\}
$$

\section{Computational strategy}

\subsection{Reduced basis and supremizer}

In the offline phase, we have to build up our reduced space $W_{N}$. The selection of the basis functions is performed by an optimal sampling algorithm which at each steps identifies a new parameter $\boldsymbol{\mu}$. Then a finite element problem has to be solved for the new selected candidate $\boldsymbol{\mu}$ to get $\boldsymbol{u}(\boldsymbol{\mu})=\left(u_{1}, u_{2}, \chi, p, \lambda\right)$. In order to achieve faster convergence (cf. $[20,36]$ ), in step 4 of the algorithm described in the following Section 3.2, the solution $\boldsymbol{u}(\boldsymbol{\mu})$ is split into its physical components (except $\lambda$, which is ignored). These are then added to the reduced space $W_{N}$.

To ensure solvability of the reduced system we add the constant pressure $(0,0,0,1,0)$ and Lagrange multiplier $(0,0,0,0,1)$ to $W_{N}$. To ensure stability, to each pressure mode in $W_{N}$, we add a supremizer $\sigma(\boldsymbol{u}(\boldsymbol{\mu})) \in X$ (cf. [26,31]). The supremizer is defined by a null temperature, pressure, and Lagrange multiplier, and as the solution of the problem

$$
(\boldsymbol{\sigma}(\boldsymbol{u}(\boldsymbol{\mu})), \boldsymbol{v})_{X}=\int_{\Omega} p \partial_{i} v_{i} \quad \forall \boldsymbol{v} \in X
$$

Note that there are alternative definitions of the supremizer, cf. [31,24,27,29].

\subsection{Offline algorithm}

The offline algorithm ([3], section 5) includes the computation of the reduced basis ingredients - optimal selection of the basis, computation of matrices and vectors in the reduced space (cf., e.g., $[30,21]$ ) — and of the a posteriori error estimation ingredients [35] - selection of $\mathcal{V}^{K}=\left\{\overline{\boldsymbol{\mu}}_{1}, \ldots, \overline{\boldsymbol{\mu}}_{K}\right\}$, solution of the eigenvalue problems ([3], section 6.4), and computation of the Sobolev embedding constants ([3], section 6.5). Because of the presence of the reduced basis approximations $\boldsymbol{u}_{\bar{N}}(\overline{\boldsymbol{\mu}})$ in the definition of the natural norms, these two stages are dependent from each other.

As preliminary step, we compute $\rho_{X}$ and we provide surrogates for $\bar{B}_{\overline{\boldsymbol{\mu}}}^{L B}(\overline{\boldsymbol{\mu}})$ and $\rho_{\overline{\boldsymbol{\mu}}}, \overline{\boldsymbol{\mu}} \in \mathcal{V}^{K}$, in order to have an error bound approximation. We expect 
that $\bar{B}_{\overline{\boldsymbol{\mu}}}^{L B}(\overline{\boldsymbol{\mu}})$ is of order one, so we set its surrogate to $\beta_{0}<1$, say, 0.2 . To compute a surrogate $\rho_{\overline{\boldsymbol{\mu}}}(\overline{\boldsymbol{\mu}})$, we select just one representative $\overline{\boldsymbol{\mu}}$ in $\mathcal{V}^{K}$ and replace $\boldsymbol{u}_{\bar{N}}(\overline{\boldsymbol{\mu}})$ by $\boldsymbol{u}(\overline{\boldsymbol{\mu}})$ in $(10)^{2}$ (hence in (13)); the value found will serve as a surrogate for $\rho_{\overline{\boldsymbol{\mu}}}(\overline{\boldsymbol{\mu}})$ for all the elements in $\mathcal{V}^{K}$. As a result we have an efficient — online fast — tool to approximately compute the error for a given $\boldsymbol{\mu}$ in $\mathcal{D}$.

We are then ready to start our offline algorithm. The optimal ("greedy") process for the selection of the basis functions of $W_{N}$ consists in the steps 3 to 5 ; in step 6 we solve for the generalized eigenvalue problems described in Section 2.6. In steps a and 7 we are required to solve a reduced basis problem (with an "intermediate" space $W_{N}$ ); this is done by applying the online algorithm described in the following section.

1. Manually set $K$ and a representative set $\mathcal{V}^{K}=\left\{\overline{\boldsymbol{\mu}}_{1}, \ldots, \overline{\boldsymbol{\mu}}_{K}\right\}$; compute $\rho_{X}$ and a surrogate for $\rho_{\overline{\boldsymbol{\mu}}}$;

2. start an optimal search algorithm: for a large random ${ }^{3}$ set of $\boldsymbol{\mu}$ 's

a) solve the reduced basis problem with the existing basis;

b) select the "nearest" $\overline{\boldsymbol{\mu}}$ in $\mathcal{V}^{K}$ and compute $\epsilon_{N}(\boldsymbol{\mu}), \tau_{N, \bar{\mu}}(\boldsymbol{\mu})$, and $\Delta_{N, \overline{\boldsymbol{\mu}}}(\boldsymbol{\mu})$ by replacing $\rho_{\overline{\boldsymbol{\mu}}}$ and $\beta_{\overline{\boldsymbol{\mu}}}(\boldsymbol{\mu})$ by their surrogates;

c) select the optimal $\boldsymbol{\mu}$ as follows

(i) if for some $\boldsymbol{\mu}$ 's, (a) did not converge, select the "furthest" from the previously selected ones;

(ii) if for some $\boldsymbol{\mu}^{\prime} \mathrm{s}, \tau_{N, \overline{\boldsymbol{\mu}}}(\boldsymbol{\mu})$ is bigger than 1, select the one with the largest $\tau_{N, \overline{\boldsymbol{\mu}}}(\boldsymbol{\mu})$

(iii) otherwise select the one with the largest $\Delta_{N, \overline{\boldsymbol{\mu}}}(\boldsymbol{\mu})$.

3. solve the finite element problem (3) for the selected $\boldsymbol{\mu}$;

4. increase $N$ and enrich the reduced basis space $W_{N}$ with $\boldsymbol{u}(\boldsymbol{\mu})$. This step involves Gramm-Schmidt orthogonalization [21] and the computation of the matrix and scalar products necessary for the reduced basis matrices and the online computation of the $Y$-dual norm of the residual (cf. [23,35]). In our model problems, $\boldsymbol{u}(\boldsymbol{\mu})$ is split into its physical components and a stabilizing supremizer is computed; the reduced space is hence enriched by four functions (cf. Section 3.1): three basis functions $\left(u_{1}, u_{2}, 0,0,0\right)$, $(0,0, \chi, 0,0),(0,0,0, p, 0)$, representing velocity, temperature and pressure, respectively, as well as the supremizer $\boldsymbol{\sigma}(\boldsymbol{u}(\boldsymbol{\mu}))$, are added to our reduced basis space. The total dimension of the reduced basis space is given by $2+4 \cdot N$ (the preliminary constant pressure, the Lagrange multiplier, $N$ velocity solutions, $N$ temperature solutions, $N$ pressure solutions and $N$ computed supremizers);

5. if the maximum number of basis desired or the tolerance requested for $\Delta_{N, \overline{\boldsymbol{\mu}}}(\boldsymbol{\mu})$ are achieved, go to 6 , otherwise go to $3 ;$

$\overline{2}$ In other words, in this first step we do not need the reduced basis space $W_{N}$.

3 This can be replaced by a deterministic search over a large set of sample points. 
6. set $\bar{N}$ to the reached number of basis functions and compute all the ingredients for the construction of $\bar{B}_{\overline{\boldsymbol{\mu}}}^{L B}(\overline{\boldsymbol{\mu}})$;

7. start a random process: for a large set of $\boldsymbol{\mu}$ 's check that the reduced basis problem can be solved, that $\bar{B}_{\overline{\boldsymbol{\mu}}}^{L B}(\boldsymbol{\mu})$ is positive for at least one $\overline{\boldsymbol{\mu}}$ and that $\tau_{N, \overline{\boldsymbol{\mu}}}(\boldsymbol{\mu}) \leq 1$ for this $\overline{\boldsymbol{\mu}}$.

If this is ok, then all the online components are ready and we set $N_{\max }=$ $N$, otherwise either add some $\overline{\boldsymbol{\mu}}$ 's to $\mathcal{V}^{K}$ and go to 6 , or go to 3 and replace $\beta_{\overline{\boldsymbol{\mu}}}(\boldsymbol{\mu})$ by $\bar{B}_{\overline{\boldsymbol{\mu}}}^{L B}(\boldsymbol{\mu})$ instead of by $\beta_{0}$.

The computational effort is largely dependent on $\mathcal{N}$ and $N_{\max }$. In the offline stage, beside $N_{\max }$ finite element solutions, we have to compute "finite element" matrix and scalar products and solve $2 K\left(Q_{0}+Q_{1}\right)$ "finite element" generalized eigenvalue problems.

\subsection{Online algorithm}

The online procedure, for given $\boldsymbol{\mu} \in \mathcal{D}$ and $N \leq N_{\max }$, reads

I. solve the reduced basis problem (8). We solve this by a Newton algorithm; as initial guess we take a solution at a nearby parameter used to generate the reduced basis. Compute $s_{N}(\boldsymbol{\mu})=\ell(\boldsymbol{u}(\boldsymbol{\mu}))$;

II. compute $\epsilon_{N}(\boldsymbol{\mu})$;

III. order $\mathcal{V}^{K}$ in increasing distance ${ }^{4}$ from $\boldsymbol{\mu}$. Compute $\bar{B}_{\overline{\boldsymbol{\mu}}}^{L B}(\boldsymbol{\mu})$ and $\tau_{N, \overline{\boldsymbol{\mu}}}(\boldsymbol{\mu})$ until we find $\overline{\boldsymbol{\mu}}$ in $\mathcal{V}$ such that $\bar{B}_{\overline{\boldsymbol{\mu}}}^{L B}(\boldsymbol{\mu})>0$ and $\tau_{N, \overline{\boldsymbol{\mu}}}(\boldsymbol{\mu}) \leq 1$. We set $\mathcal{I}^{K}(\boldsymbol{\mu})=\overline{\boldsymbol{\mu}}$

IV. compute $\Delta_{N, \overline{\boldsymbol{\mu}}}(\boldsymbol{\mu})$ and $\Delta_{N, \overline{\boldsymbol{\mu}}}^{s}(\boldsymbol{\mu})$.

If in step 4 . we do not find any valid $\overline{\boldsymbol{\mu}}$, our method fails: we can not provide an error bound nor even existence; if $N \leq N_{\max }$, then $N$ must be increased, otherwise our basis is not rich enough.

The computational effort is independent of $\mathcal{N}$ : step I has dominant complexity $N^{3}+Q_{1} N^{2}$ times the number of Newton iterations required, while step II has complexity $Q_{1}^{2} N^{4}+Q_{0} Q_{1} N^{3}$. The complexity of steps 4 and IV is dominated by that of step II.

$\overline{4}$ The type of distance function is not much relevant. 


\section{Numerical results}

\subsection{Finite element solver}

We implemented a parallel finite element solver that uses the Trilinos ${ }^{5}$ library, in particular Epetra as MPI interface, Amesos [34] as dense direct solver, Aztec00 as linear iterative solver with domain decomposition preconditioners from IFPACK [33], and Anasazi as eigenvalue solver, in particular LOBPCG $[13]$.

We used Taylor-Hood P2 (velocity, temperature)/P1 (pressure) finite elements for a total of 38000 degrees of freedom. We modified a preconditioner proposed in [4]: at a point $\boldsymbol{u} \in X$, we define $P^{0}$ as $d a(\boldsymbol{u}, \boldsymbol{\mu})$ where the divergence operator is replaced by the pressure mass matrix on the pressure block diagonal. We then construct a one-level Schwarz preconditioner $P[33,32]$ to $P^{0}$ and perform a local $L U$ factorization. We solve the Jacobian system with restarted PGMRES(500) with preconditioner $P$. The resulting operator $P$, which depends on $\boldsymbol{u}$ and $\boldsymbol{\mu}$, is fast, distributed, and is an effective preconditioner for the problem up to $\mathrm{Gr}=10^{7}$.

We are interested in one solution branch, therefore we use homotopy with respect to the parameters when solving the finite element problem. Without homothopy, the finite element problem does not converge. In contrast, in our examples, the reduced basis problem does not need homotopy: our initial guess is a known solution for a nearby parameter (a solution that has generated our reduced space).

In Figure 2 we present the streamlines for increasing Grashof number ( $\mathrm{Gr}=$ $\left.5 \cdot 10^{4}, 6.125 \cdot 10^{4}, 7.5 \cdot 10^{4}\right)$ and different aspect ratio $(A=1,1.125,1.25)$ and $\operatorname{Pr}=7$. We note the presence of an asymmetric convective cell and a thermal boundary layer against the heated wall.

In Table 1 we report the computational time needed to complete the offline part of our method and also the time to find the finite element solutions for 9 different parameter instances. The offline part takes much more time than just some finite element resolutions; the advantage of the reduced basis, resides on the offline/online splitting. In fact, once the ingredients are ready and given a parameter instance, the resolution of the reduced basis problem and the computation of a rigorous error bound takes less than a tenth of a second on a single processor notebook to provide a certified and reliable solution (cf. Figures 4, 7, and 10).

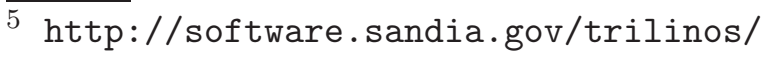



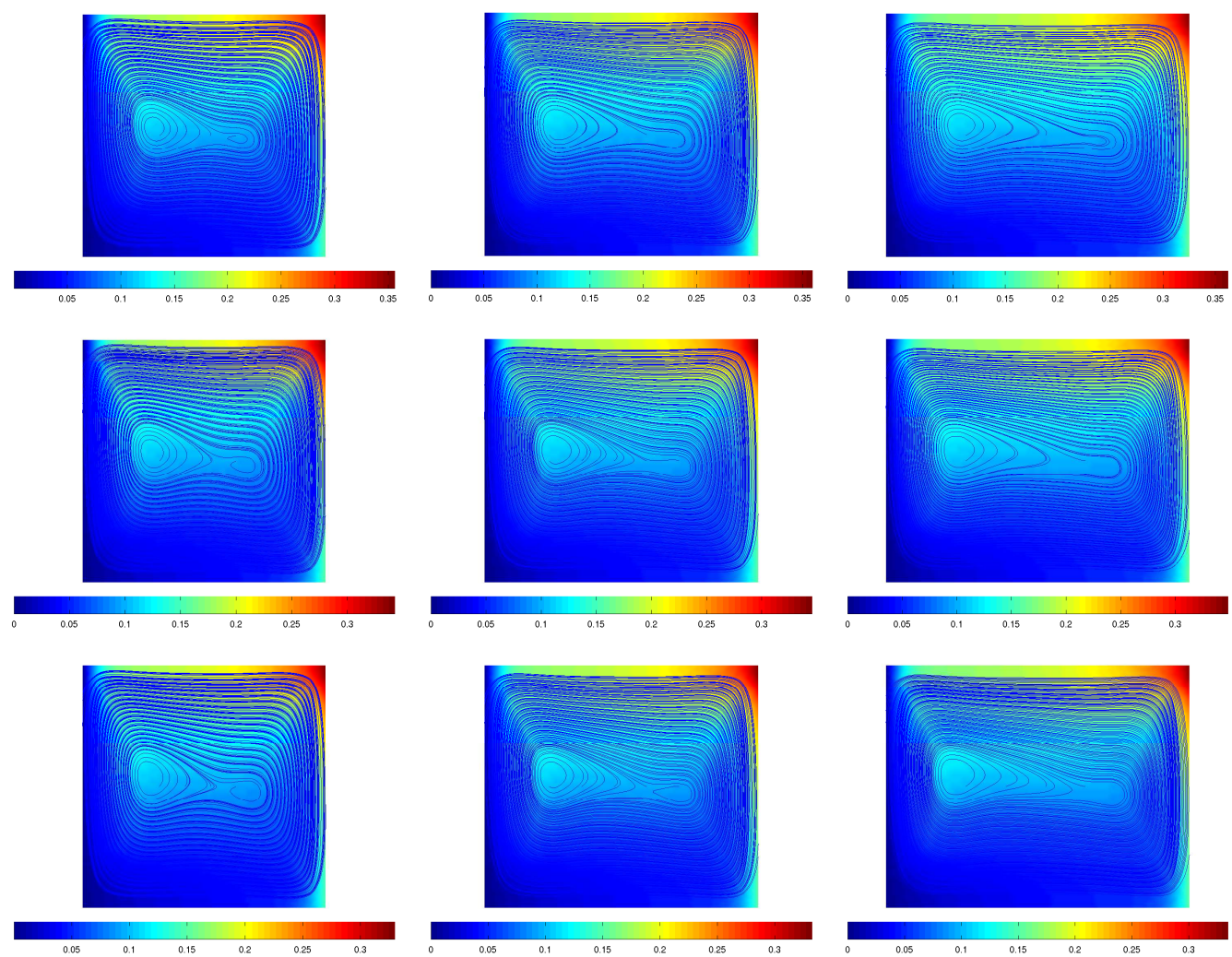

Fig. 2. Streamlines at Grashof numbers $\mathrm{Gr}=5 \cdot 10^{4}, 6.125 \cdot 10^{4}, 7.5 \cdot 10^{4}$, (first, second, third row), Prandtl number $\operatorname{Pr}=7$, and aspect ratio of the cavity $A=1,1.125,1.25$ (first, second, third column).

Table 1

\begin{tabular}{|c|c|c||c|}
\hline & offline RB $\left(N_{\max }\right)$ & offline $\bar{B}_{\overline{\boldsymbol{\mu}}}^{L B}(\# \overline{\boldsymbol{\mu}})$ & $\mathrm{FE}(\# \boldsymbol{\mu})$ \\
\hline Gr-Pr & 1h44' $(12)$ & $27 \mathrm{~h} 15^{\prime}(21)$ & $30^{\prime}(9)$ \\
$\mathrm{Gr}-\mathrm{A}$ & 11h24' (18) & $41 \mathrm{~h} 48^{\prime}(22)$ & $23^{\prime}(9)$ \\
$\mathrm{Pr}-\mathrm{A}$ & 13h00' $(18)$ & 41h41' $(20)+36 \mathrm{~h} 40^{\prime}(11)$ & $1 \mathrm{~h} 04^{\prime}(9)$ \\
\hline
\end{tabular}

Wall times on a 16 nodes cluster; "offline RB": computation of the reduced basis ingredients (steps 3 to 5 in the offline algorithm), $N_{\max }$ is the number of selected $\boldsymbol{\mu}$ 's; "offline $\bar{B}_{\bar{\mu}}^{L B}$ ": resolution of the eigenvalue problems (step 6 in the offline algorithm), the number between brackets is the number of selected $\overline{\boldsymbol{\mu}}$ 's; "FE": solution of the finite element problems for some instances of the parameters, the number between brackets is the number of instances.

\subsection{Reduced basis resolution and error bounds}

Before introducing the numerical results for different combinations of variable parameters, we show here the "visual" convergence of the reduced basis approximation to the reference finite element solution. We report in Figure 3, as example, the reduced basis solution with streamlines at Grashof number 
$\mathrm{Gr}=10^{5}$, Prandtl number $\operatorname{Pr}=1$, and aspect ratio $A=1$ for $N=2,3,4,5,6$ and the comparison with the reference finite element solution. We can see the fast convergence of the method and how the physical solution provided by reduced basis is approaching the reference one. For $N=6$ the reduced basis solution is already a very good approximation for the finite element one. However, this is not satisfying, since in this case for $N=6$ we have that $\tau_{N, \bar{\mu}}(\boldsymbol{\mu})>1$, which means that we do not have any guarantee that a reference finite element solution exists nearby the reduced basis approximation.
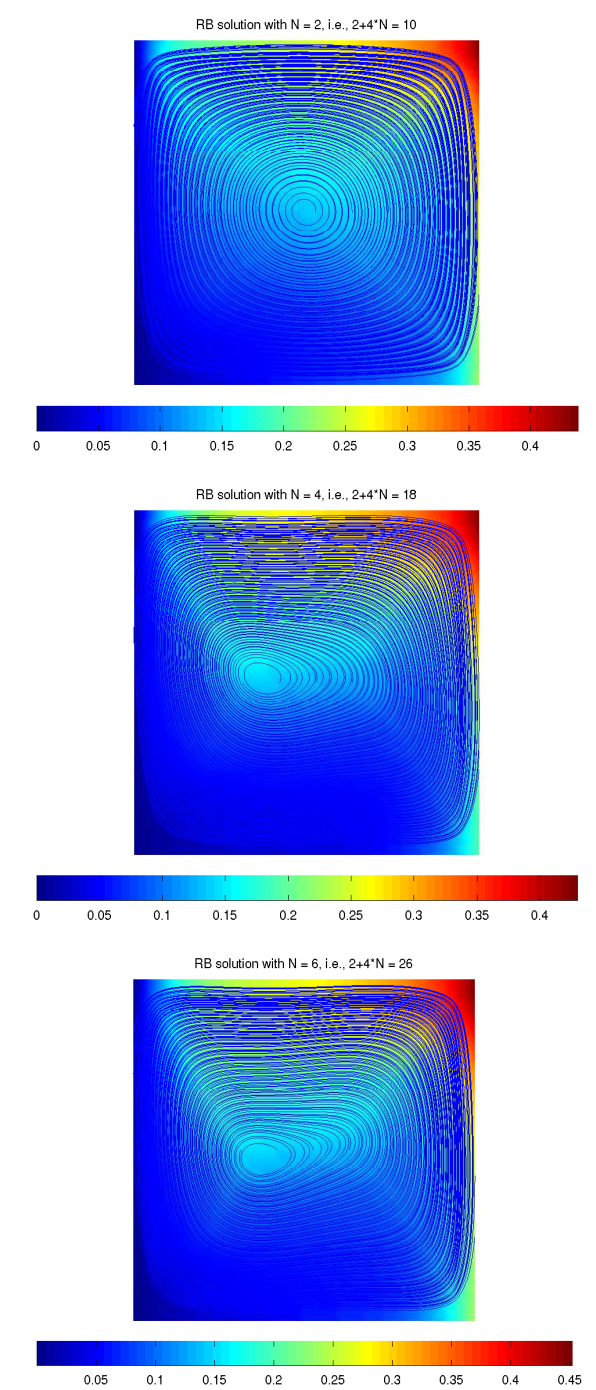

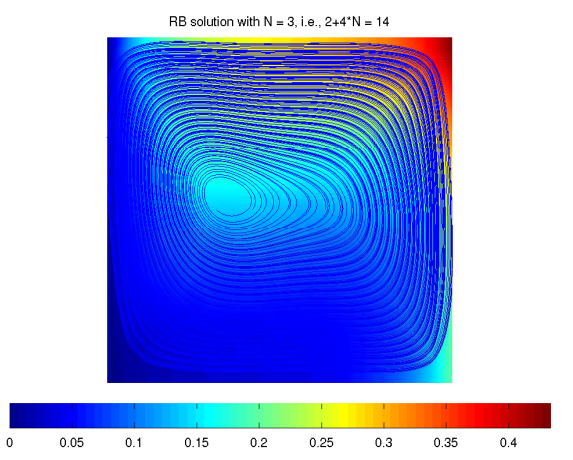

RB solution with $\mathrm{N}=5$, i.e., $2+4^{*} \mathrm{~N}=22$
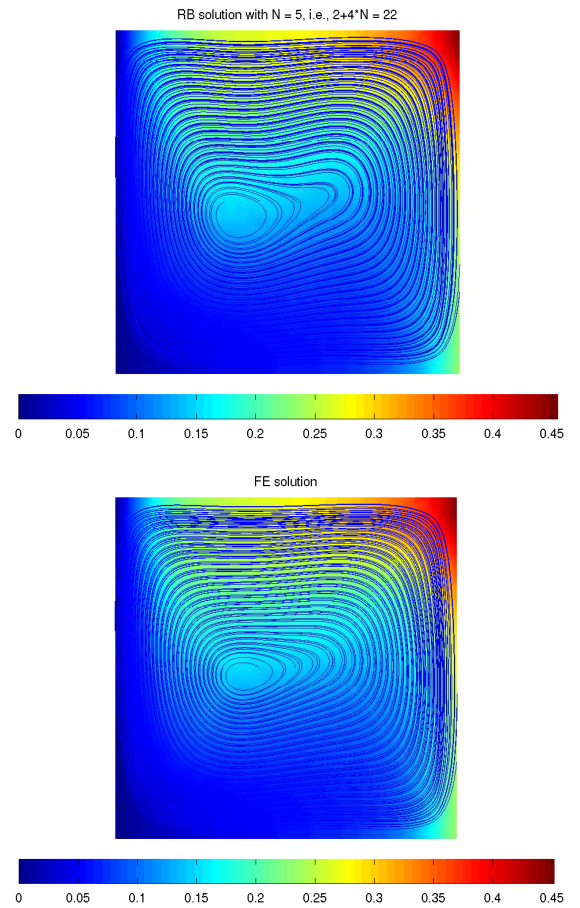

Fig. 3. Streamlines at Grashof number $\mathrm{Gr}=10^{5}$, Prandtl number $\operatorname{Pr}=1$, and aspect ratio $A=1$ : streamlines of the reduced basis solution for $N=2,3,4,5,6$ and of the reference finite element solution.

In the following subsections we provide some numerical results for the proposed methodology combining each time two different parameters: Grashof-Prandtl, Grashof-aspect ratio and Prandtl-aspect ratio by reporting computational times and some ingredients used to compute error bounds on the solution and 
on the outputs.

\subsubsection{Case $\mathrm{Gr}-\mathrm{Pr}$}

In Figure 4 we report the convergence rate and CPU time comparison over 1000 random samples (different for different $N$ ) for the case with Grashof and Prandtl numbers as parameters (cf. Section 2.2.1) in the range $\left[10^{3}, 10^{5}\right] \times$ $[0.7,7]$. We report the most pessimistic value over our samples: $\max \tau_{N, \bar{\mu}}(\boldsymbol{\mu})>$ 1 means that we do not have an error estimate for every sample. $\Delta_{(1), N}^{s}$ and $\Delta_{(2), N}^{s}$ are the output error bounds referring to the two different outputs for the samples tested, $\Delta_{N}$ is the error bound related to the field variables. Their maxima are taken over the samples we can provide existence and unicity for (i.e., $\beta_{\overline{\boldsymbol{\mu}}}(\boldsymbol{\mu})>0$ and $\tau_{N, \overline{\boldsymbol{\mu}}}(\boldsymbol{\mu})>1$ ). RB[ms] counts the average CPU time for solving the reduced basis problem and $\Delta[\mathrm{ms}]$ the one for the error bound computation (dual norm of the residual and inf-sup lower bounds). Note that $\mathrm{RB}[\mathrm{ms}]$ is of order $N^{3}$, while $\Delta[m s]$ is of order $N^{4}$, which explains the behavior in Figure 4 (cf. end of Section 3.3).

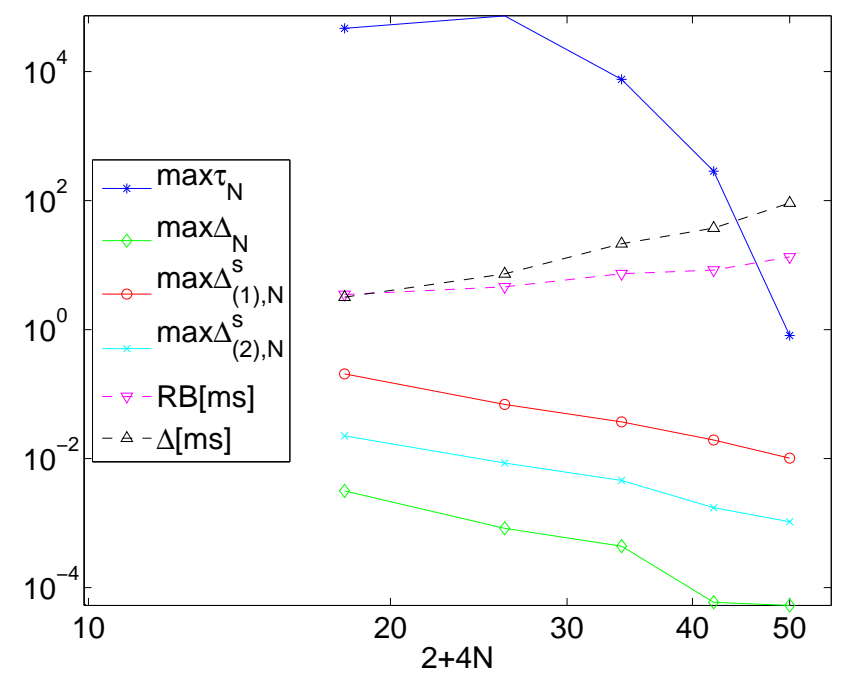

Fig. 4. Grashof and Prandtl numbers as parameters in the range $\left[10^{3}, 10^{5}\right] \times[0.7,7]$. Convergence rate and CPU time comparison over 1000 random samples. $\Delta_{(1), N}^{s}$ and $\Delta_{(2), N}^{s}$ are the output error bounds referring to the two different outputs for the samples tested, $\Delta_{N}$ is the error bound on the solution. $\mathrm{RB}[\mathrm{ms}]$ counts the average $\mathrm{CPU}$ time for solving the reduced basis and $\Delta[\mathrm{ms}]$ the CPU time for the error bound (dual norm of the residual and inf-sup lower bounds).

Figures 5 and 6 show the values of the quantities related to the error bounds with a reduced basis approximation with a total dimension of $2+4 \cdot N$, where $N=12$. In Figure 5 we report upper left the inf-sup lower bound $\bar{B}_{\overline{\boldsymbol{\mu}}}^{L B}(\boldsymbol{\mu})$. The small hole represents a region where our lower bound is negative. Where $\bar{B}_{\overline{\boldsymbol{\mu}}}^{L B}(\boldsymbol{\mu})=1$ we also recognize the elements of $\mathcal{V}^{K}=\left\{\overline{\boldsymbol{\mu}}_{1}, \ldots, \overline{\boldsymbol{\mu}}_{K}\right\}$ (here $K=21)$. Upper right we report $\tau_{\overline{\boldsymbol{\mu}}}(\boldsymbol{\mu})$ and lower left the upper bound $\Delta_{N, \overline{\boldsymbol{\mu}}}(\boldsymbol{\mu})$ 
to the natural norm of the error in the field variable. Lower left we recognize two holes where we do not have an error estimate: the first one is related to the negative $\bar{B}_{\overline{\boldsymbol{\mu}}}^{L B}(\boldsymbol{\mu})$, the second one to $\tau_{\overline{\boldsymbol{\mu}}}(\boldsymbol{\mu})>1$ and in particular to $\bar{B}_{\overline{\boldsymbol{\mu}}}^{L B}(\boldsymbol{\mu})$ close to zero (the dual norm of the residual is actually already small). To fill these holes we should therefore add two $\overline{\boldsymbol{\mu}}$ in the middle to provide a complete coverage in our parameter space (cf. $\mathrm{Pr}-\mathrm{A}$ case). Lower right we report the effectivity of our error upper bound: $\Delta_{N, \overline{\boldsymbol{\mu}}}(\boldsymbol{\mu}) /\left\|u(\boldsymbol{\mu})-u_{N}(\boldsymbol{\mu})\right\|_{\overline{\boldsymbol{\mu}}}$; here, only for comparison, we have computed the finite element solution for each random parameter. The error bounds are small and, also important, the effectivities are small thanks to our natural norms approach (cf. (22)). Note how the error bounds are closely related to the parameters that generate our reduced basis, while the effectivities are closely related to the inf-sup lower bound. Since our inf-sup lower bound is better "nearby a $\overline{\boldsymbol{\mu}}$ " the effectivity is better "nearby a $\bar{\mu} "$.

In Figure 6 we report upper left the output $s_{(1)}(\boldsymbol{\mu})$ as function of the Grashof and Prandtl numbers, upper right the computed error $\left|s_{(1)}(\boldsymbol{\mu})-s_{(1), N}(\boldsymbol{\mu})\right|$ on the first output, and lower left the upper bound to error for the same output $\Delta_{N, \bar{\mu}}^{s}(\boldsymbol{\mu})$. Lower right we report the effectivity of our error upper bound, $\Delta_{N, \bar{\mu}}^{s}(\boldsymbol{\mu}) /\left|s_{(1)}(\boldsymbol{\mu})-s_{(1), N}(\boldsymbol{\mu})\right|$. Note that the error bound is always smaller than $8 \cdot 10^{-3}$ and that the effectivities for the first output are less sharp than for the field variable (the second output leads to similar results). However the worse effectivities appear when the real error is very small, otherwise when the real error is "large" the effectivities are good.

\subsubsection{Case $G r-A$}

We report the equivalent figures as for the $\mathrm{Gr}-\mathrm{Pr}$ case, here we describe only the differences. The parameters are the Grashof number and the aspect ratio of the cavity (cf. Section 2.2.2) in the range $\left[5 \cdot 10^{4}, 7.5 \cdot 10^{4}\right] \times[1.25,1.5]$ and $\mathcal{V}^{K}$ with 22 elements. Figure 7 shows the convergence rate and the online CPU time with respect to the number of reduced basis used.

Figures 8 and 9 show the values of the quantities related to the error bounds with a reduced basis approximation with a total dimension of $2+4 \cdot N$, where $N=18$.

In this example (cf. Figure 8), the inf-sup lower bound is always positive and $\tau_{\bar{\mu}}(\boldsymbol{\mu})$ smaller or equal to one. We are therefore able to provide an error estimate for all the 1000 parameters that we have tested. In Figure 9 we report the results concerning the first output. 

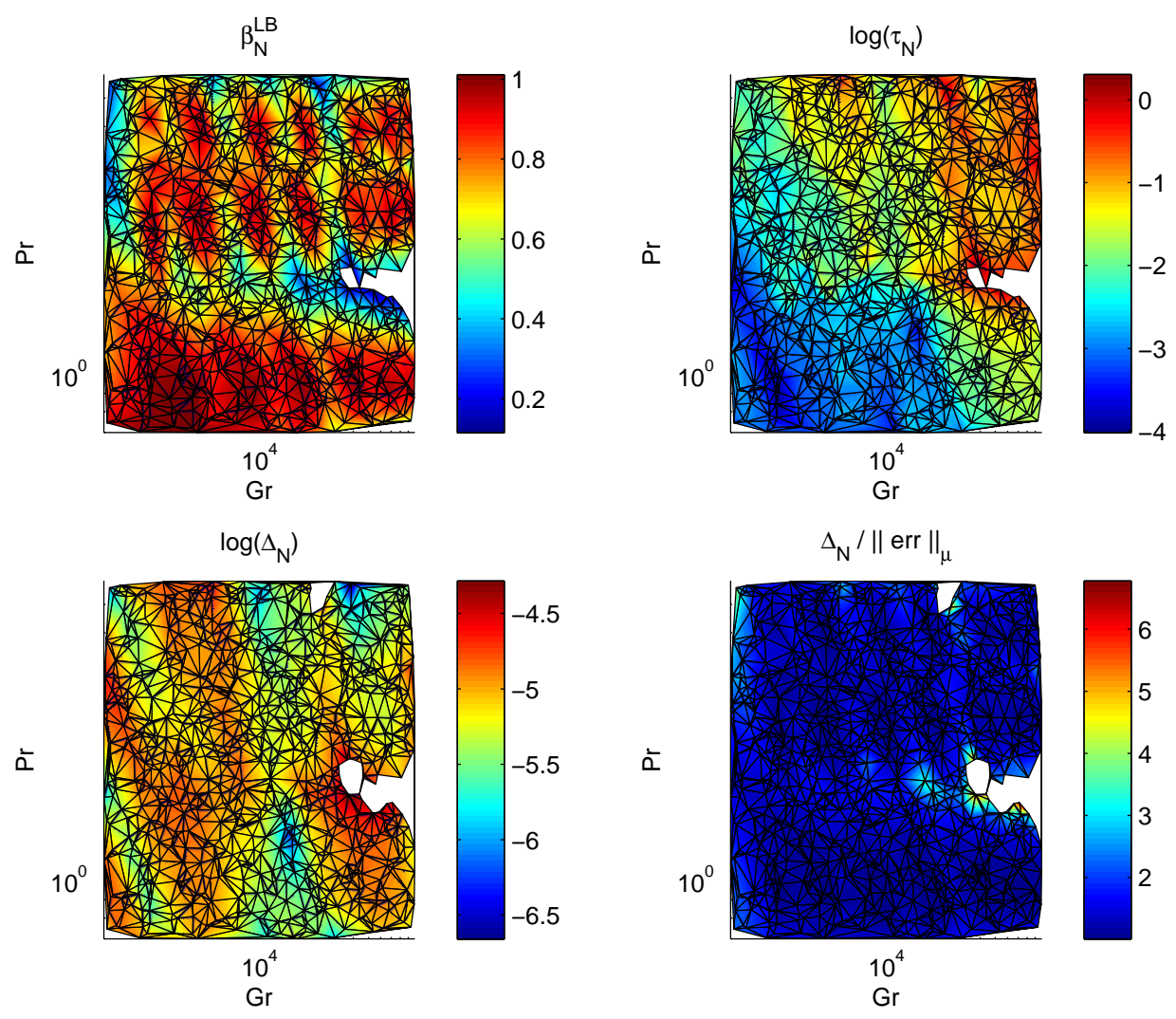

Fig. 5. Two physical parameters: Grashof and Prandtl numbers in the range $\left[10^{3}, 10^{5}\right] \times[0,7]$. The $x$-axis represents the Grashof number and the $y$-axis the Prandtl number, both in logarithmic scale. Upper left: inf-sup lower bound $\bar{B}_{\overline{\boldsymbol{\mu}}}^{L B}(\boldsymbol{\mu})$ (the small hole represents a region where our lower bound is negative, and therefore useless. One or more $\overline{\boldsymbol{\mu}}$ should be added in the zone). Upper right: $\tau_{\overline{\boldsymbol{\mu}}}(\boldsymbol{\mu})$. Lower right: upper error bound $\Delta_{N, \overline{\boldsymbol{\mu}}}(\boldsymbol{\mu})$ on the field variable. In the small hole we are not providing any error bound (left for demonstrative purposes). Lower left: effectivity on the field variable error w.r.t. the natural norm $\Delta_{N, \overline{\boldsymbol{\mu}}}(\boldsymbol{\mu}) /\left\|u(\boldsymbol{\mu})-u_{N}(\boldsymbol{\mu})\right\|_{\overline{\boldsymbol{\mu}}}$.

\subsubsection{Case $\operatorname{Pr}-A$}

The parameters are the Prandtl number and the aspect ratio of the cavity (cf. Section 2.2.3) in the range $[1,7] \times[1.25,1.5]$. Figure 10 shows the convergence rate and the online $\mathrm{CPU}$ time with respect to the number of reduced basis used. Figures 11, 12, and 13 show the values of the quantities related to the error bounds given by a reduced basis approximation with a total dimension of $2+4 N$, where $N=18$. We first take $\mathcal{V}^{K}$ with 20 elements (Figure 11); for several parameter values we are not able to provide a positive inf-sup lower bound (the holes represent a region where our lower bounds are negative, and therefore useless). Therefore we complete $\mathcal{V}^{K}$ with other 11 elements (Figure 12), this allows to compute an error bound for (almost) all the parameters in $\mathcal{D}$. Where our lower bound is still negative one or more $\overline{\boldsymbol{\mu}}$ 's should be added in the corresponding zones. 

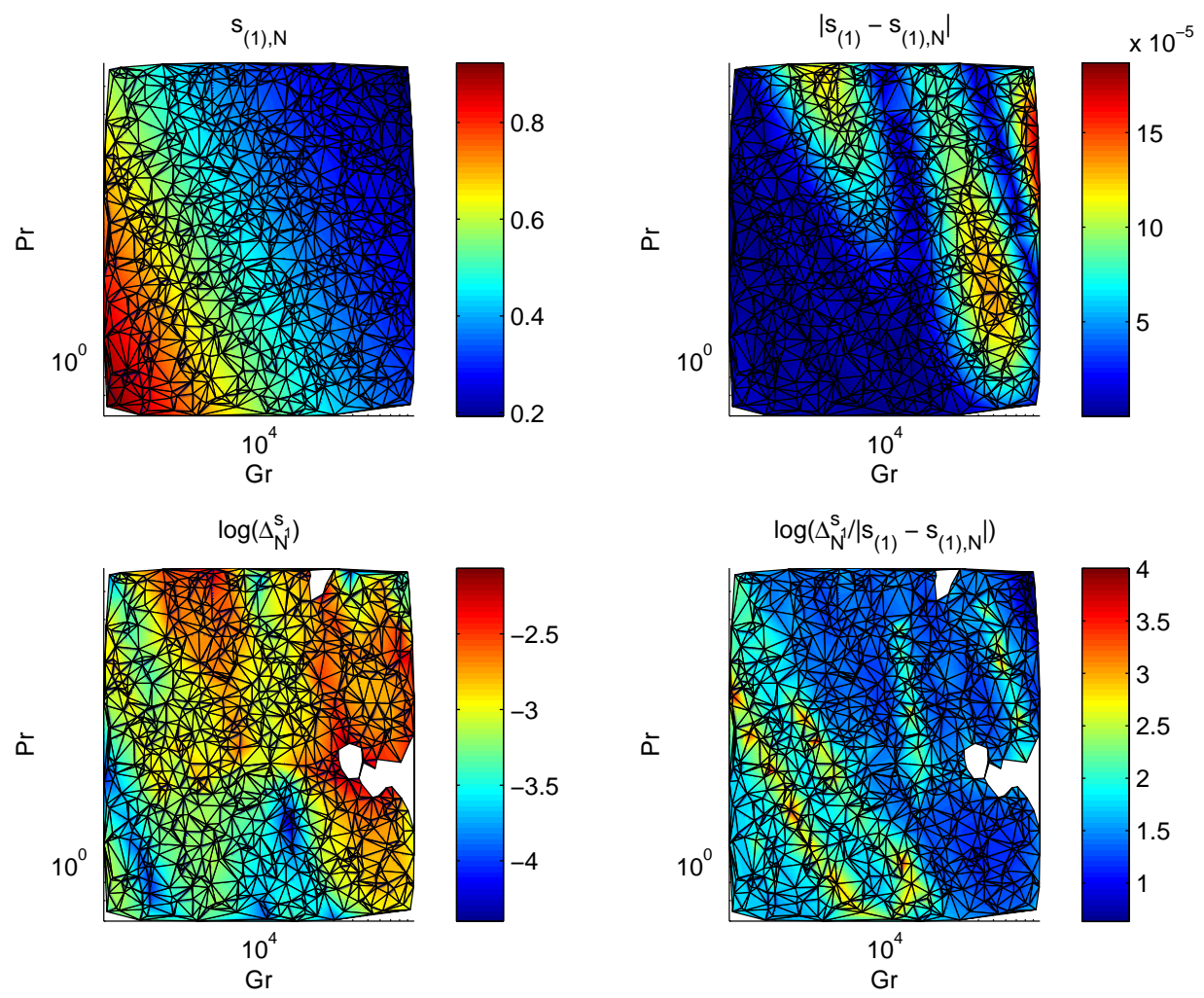

Fig. 6. Two physical parameters: Grashof and Prandtl numbers in the range $\left[10^{3}, 10^{5}\right] \times[0.7,7]$. The $x$-axis represents the Grashof number and the $y$-axis the Prandtl number, both in logarithmic scale. Upper left: output $s_{(1)}$. Upper right: real error $\left|s_{(1)}(\boldsymbol{\mu})-s_{(1), N}(\boldsymbol{\mu})\right|$ between finite element and reduced basis solutions. Lower left: upper error bound on for the output $\Delta_{N, \bar{\mu}}^{s}(\boldsymbol{\mu})$. Lower right.: logarithm of the effectivity $\Delta_{N, \bar{\mu}}^{s}(\boldsymbol{\mu}) /\left|s_{(1)}(\boldsymbol{\mu})-s_{(1), N}(\boldsymbol{\mu})\right|$.

\section{Conclusions and perspective work}

We have extended to multi-parameter dependence the numerical results proposed in $[3,20,36]$ for steady incompressible Navier-Stokes equations to describe natural-convection phenomena. We have considered the combination of Grashof and Prandtl numbers as parameters and we have included the presence of geometrical changes (with the aspect ratio of the cavity as a further parameter). The methodology is feasible: the offline CPU time is still acceptable and the online performance is not affected. The approach has proven good effectivities in the field variable error bounds and empirical good effectivities in the output error bounds.

Another aspect of interest, under investigation, is the parametric study of multiple steady states of convective flows and their stability in laterally heated cavity. The existence of multiple steady states, characterized by a different number of recirculation zones, depends on the aspect ratio of the cavity con- 


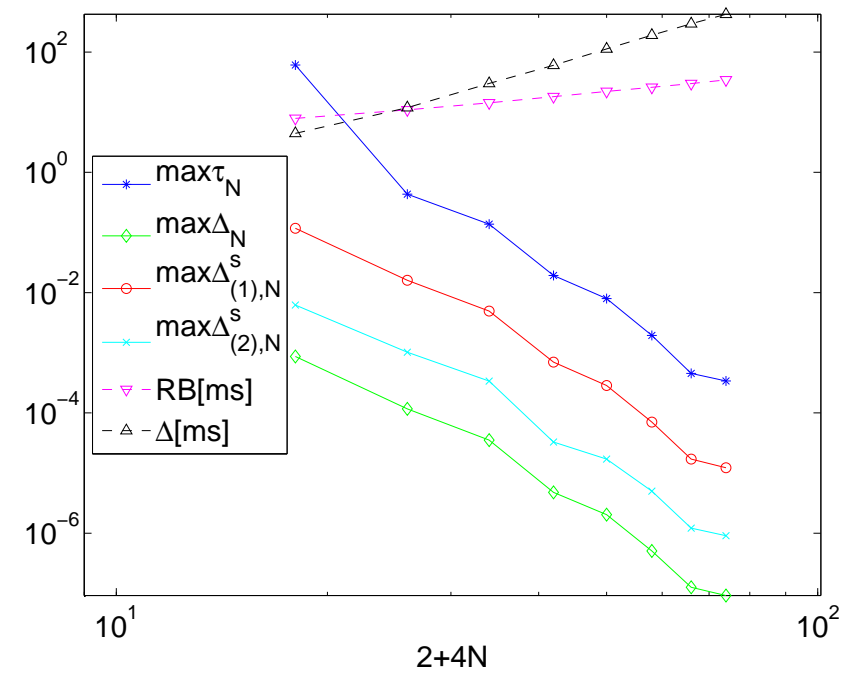

Fig. 7. Grashof and aspect ratio as parameters in the range $\left[5 \cdot 10^{4}, 7.5 \cdot 10^{4}\right] \times[1.25,1.5]$. Convergence rate and CPU time comparison over 1000 random samples. $\Delta_{(1), N}^{s}$ and $\Delta_{(2), N}^{s}$ are the output error bounds referring to the two different outputs for the samples tested, $\Delta_{N}$ is the error bound on the solution. $\tau_{N, \bar{\mu}}(\boldsymbol{\mu})$ is decreasing better than the previous case. The maximal error bounds are taken over the samples we can provide existence and unicity for. $\mathrm{RB}[\mathrm{ms}]$ counts the mean CPU time for solving the reduced basis and $\Delta[\mathrm{ms}]$ the one for the error bound (dual norm of the residual and inf-sup lower bounds).

sidered, on Prandtl and Grashof numbers (see [7]). It is our interest to build proper reduced basis approximation spaces to capture these phenomena and to study their stability. We presume that the (parameter-dependent) solution lays on an isolated branch. The natural norm approach should also allow to consider bifurcations, since the inf-sup constant is of order one with respect to the "local" norm . Nevertheless, the natural norm degenerates near the bifurcation because of a singular mode. This mode has probably to be treated by deflation [35] and the eigenvalue solver should take into account the norm degeneration.

The study of transient flows and the introduction of time as a parameter is another aspect of great interest. See the recent works $[19,18]$ where a different approach is used in the computation of the error estimators and for the inf-sup lower bound [11].

At this point one of the principal aim of this research activity is the development of numerical methods for systems optimization. We are interested in combining our computational technique built on reduced basis method with optimal control problems to solve in real-time complex optimization problems arising in fluid flow control [12]. The structure of the problem based on an input (control) - output (cost functional) relationship is well suited for combining reduced basis and optimal control techniques. Also the online-offline 

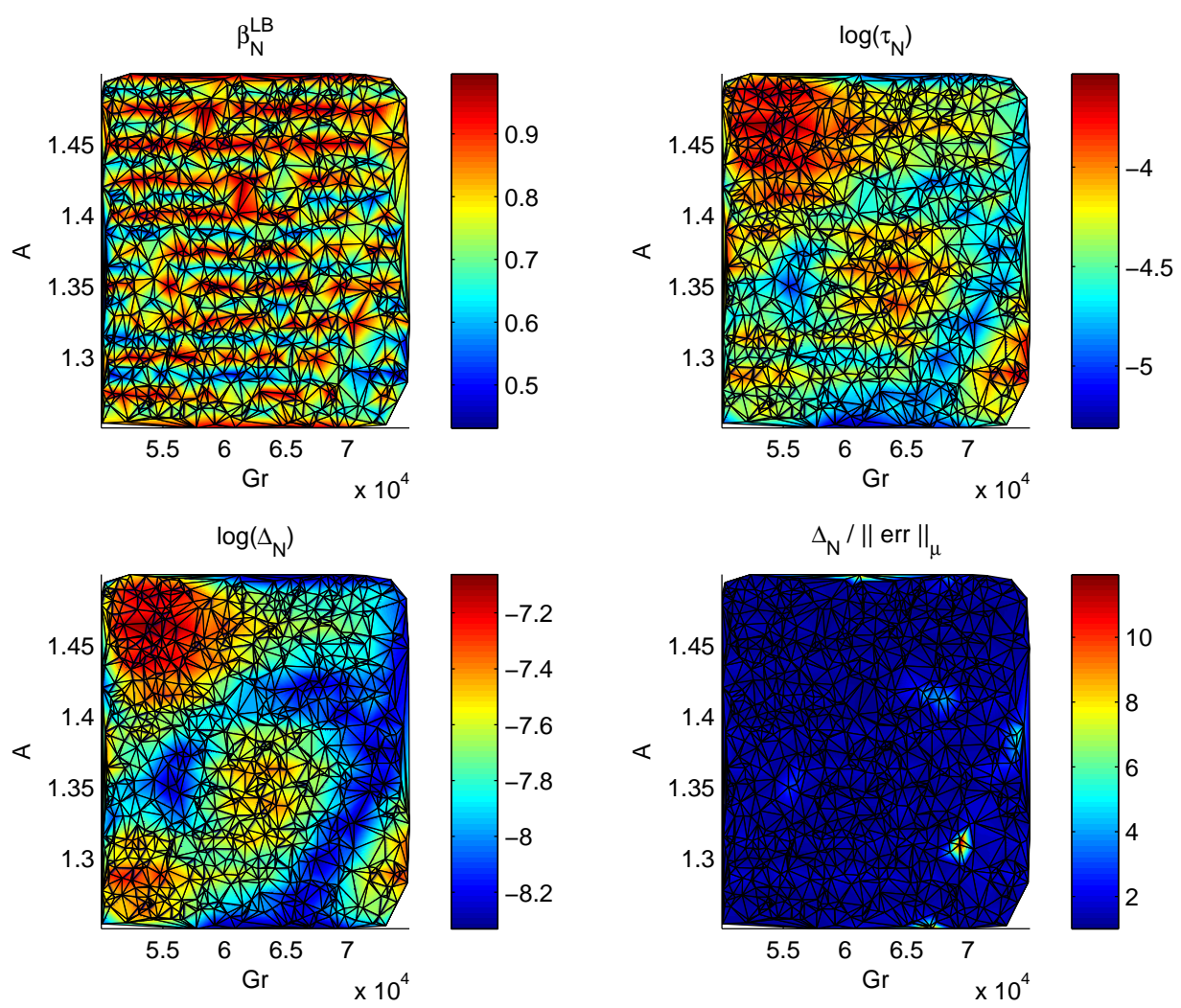

Fig. 8. One physical and one geometrical parameter: Grashof and aspect ratio in the range $\left[5 \cdot 10^{4}, 7.5 \cdot 10^{4}\right] \times[1.25,1.5]$. The $x$-axis in log scale represents the Grashof number and the $y$-axis in linear scale represents the aspect ratio. Upper left: infsup lower bound $\bar{B}_{\overline{\boldsymbol{\mu}}}^{L B}(\boldsymbol{\mu})$. Upper right: $\tau_{\overline{\boldsymbol{\mu}}}(\boldsymbol{\mu})$. Lower right: $\Delta_{N, \overline{\boldsymbol{\mu}}}(\boldsymbol{\mu})$. Lower left: effectivity on the field variable error w.r.t. the natural norm.

computational architecture allow a promising combination of reduced basis techniques with optimal control [25].

Finally, we mention another topic of current research interest: the "reduced basis element method" [14], a marriage of reduced basis and domain decomposition concepts that permits much greater geometric complexity and also provides a framework for the integration of multiple models.

\section{Acknowledgements}

We acknowledge Professor Anthony T. Patera (MIT) for his valuable comments, many contributions, insights and guidelines. We are grateful to Professor Alfio Quarteroni (EPFL) for many discussions on the subject, encouragement and comments. 

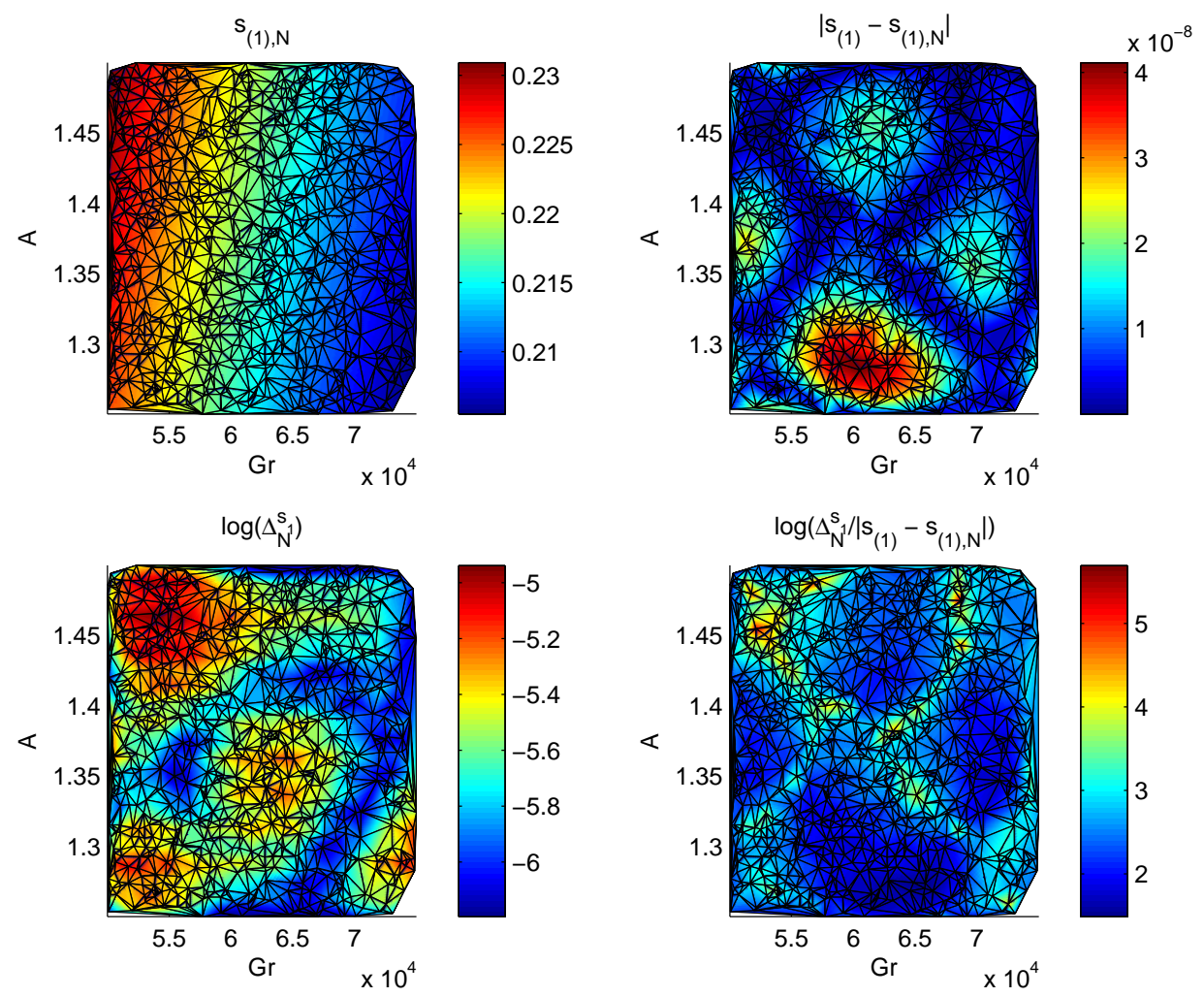

Fig. 9. One physical and one geometrical parameter: Grashof and aspect ratio in the range $\left[5 \cdot 10^{4}, 7.5 \cdot 10^{4}\right] \times[1.25,1.5]$. The $x$-axis in log scale represents the Grashof number and the $y$-axis in linear scale represents the aspect ratio. Upper left: output $s_{(1)}$. Upper right: real error $\left|s_{(1)}(\boldsymbol{\mu})-s_{(1), N}(\boldsymbol{\mu})\right|$ between finite element and reduced basis solutions. Lower left: upper bounds to error for the same output, $\Delta_{N, \bar{\mu}}^{s}(\boldsymbol{\mu})$. Lower right.: effectivity of our error upper bound, $\Delta_{N, \overline{\boldsymbol{\mu}}}^{s}(\boldsymbol{\mu}) /\left|s_{(1)}(\boldsymbol{\mu})-s_{(1), N}(\boldsymbol{\mu})\right|$.

\section{References}

[1] F. Brezzi, J. Rappaz, P. Raviart, Finite dimensional approximation of nonlinear problems. Part I: Branches of nonsingular solutions, Numerische Mathematik 36 (1980) 1-25.

[2] G. Caloz, J. Rappaz, Numerical analysis for nonlinear and bifurcation problems, in: P. Ciarlet, J. Lions (eds.), Handbook of Numerical Analysis, Vol. V, Techniques of Scientific Computing (Part 2), Elsevier Science B.V., 1997, pp. 487-637.

[3] S. Deparis, Reduced basis error bound computation of parameter-dependent navier-stokes equations by the natural norm approach, SIAM journal of numerical analysis 46 (4) (2008) 2039-2067.

[4] H. C. Elman, D. J. S., A. J. Wathen, Finite elements and fast iterative solvers: with applications in incompressible fluid dynamics, Numerical Mathematics and Scientific Computation, Oxford University Press, New York, 2005. 


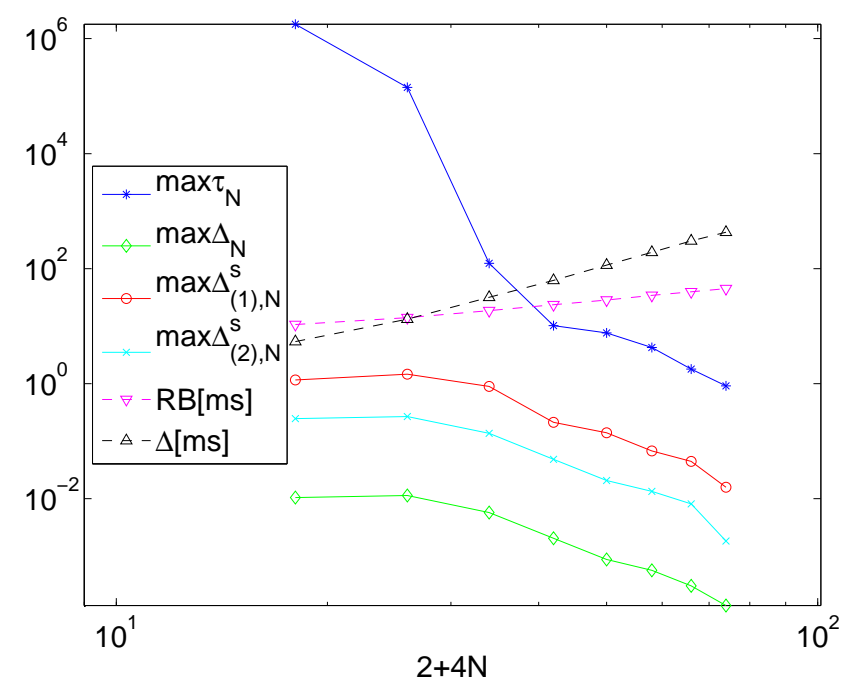

Fig. 10. Prandtl and aspect ratio as parameters in the range $[1,7] \times[1.25,1.5]$ and $\mathcal{V}^{K}=31$. Convergence rate and CPU time comparison over 1000 random samples. $\mathrm{RB}[\mathrm{ms}]$ counts the mean CPU time for solving the reduced basis and $\Delta[\mathrm{ms}]$ the one for the error bound (dual norm of the residual and inf-sup lower bounds).

[5] J. P. Fink, W. C. Rheinboldt, On the error behavior of the reduced basis technique for nonlinear finite element approximations, Z. Angew. Math. Mech. 63 (1983) 21-28.

[6] M. Fortin, F. Brezzi, Mixed and Hybrid Finite Element Methods, vol. 15 of Springer Series in Computational Mathematics, Springer Verlag, 1991.

[7] A. Y. Gelfgat, P. Z. Bar-Yoseph, A. L. Yarin, Stability of multiple steady states of convection in laterally heated cavities, Journal of Fluid Mechanics 388 (1999) $315-334$.

[8] V. Girault, P. Raviart, Finite element approximation of the Navier-Stokes equations, Springer-Verlag, 1986.

[9] M. A. Grepl, N. C. Nguyen, K. Veroy, A. T. Patera, G. R. Liu, Certified rapid solution of partial differential equations for real-time parameter estimation and optimization, in: Proceedings of the $2^{\text {nd }}$ Sandia Workshop of PDEConstrained Optimization: Towards Real-Time and On-Line PDE-Constrained Optimization, SIAM Computational Science and Engineering Book Series, 2007.

[10] M. D. Gunzburger, Finite Element Methods for Viscous Incompressible Flows: A Guide to Theory, Practice, and Algorithms, Academic Press, Boston, 1989.

[11] D. B. P. Huynh, G. Rozza, S. Sen, A. T. Patera, A successive constraint linear optimization method for lower bounds of parametric coercivity and inf-sup stability constants, C. R. Acad. Sci. Paris, Analyse Numérique 345 (8) (2007) $473-478$.

[12] K. Ito, S. S. Ravindran, A reduced-order method for simulation and control of fluid flows, Journal of Computational Physics 143 (2) (1998) 403-425. 

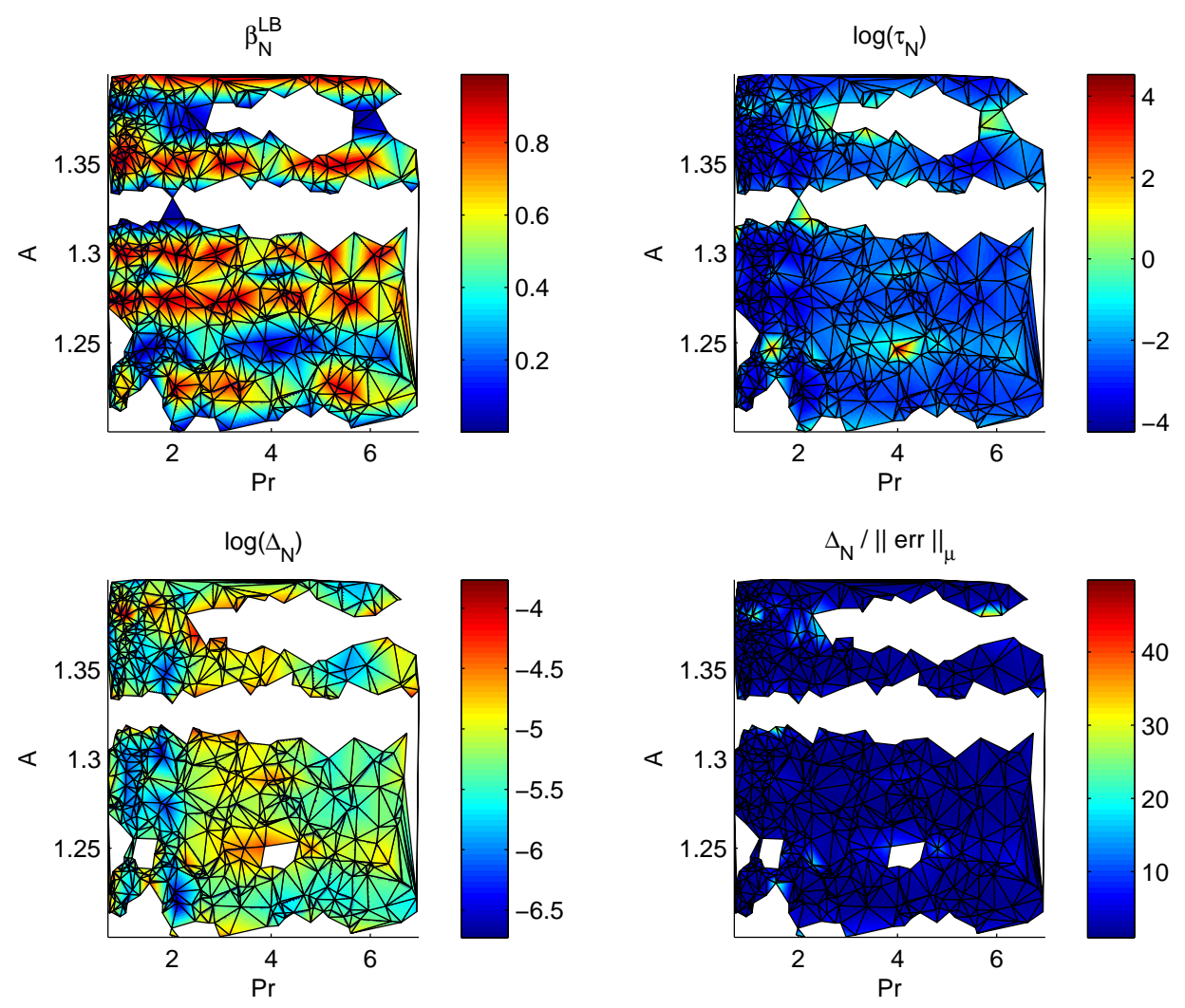

Fig. 11. One physical and one geometrical parameter: Prandtl and aspect ratio in the range $[1,7] \times[1.25,1.5]$ and $\mathcal{V}^{K}=20$. The $x$-axis represents the Prandtl number and the $y$-axis the aspect ratio, both in linear scale. Upper left: inf-sup lower bound $\bar{B}_{\overline{\boldsymbol{\mu}}}^{L B}(\boldsymbol{\mu})$. Upper right: $\tau_{\overline{\boldsymbol{\mu}}}(\boldsymbol{\mu})$. Lower right: $\Delta_{N, \overline{\boldsymbol{\mu}}}(\boldsymbol{\mu})$. Lower left: effectivity on the field variable error w.r.t. the natural norm.

[13] A. V. Knyazev, Toward the optimal preconditioned eigensolver: locally optimal block preconditioned conjugate gradient method, SIAM J. Sci. Comput. 23 (2) (2001) 517-541 (electronic), copper Mountain Conference (2000).

[14] A. Løvgren, Y. Maday, E. Rønquist, The reduced basis element method for fluid flows, in: C. Calgaro, J.-F.Coulombel, T. Goudon (eds.), Analysis and Simulation of Fluid Dynamics, vol. VII of Adv. Math. Fluid Mech., Birkhäuser, 2007, pp. 129-154.

[15] Y. Maday, A. T. Patera, D. V. Rovas, A blackbox reduced-basis output bound method for noncoercive linear problems, in: D. Cioranescu, J.-L. Lions (eds.), Nonlinear Partial Differential Equations and Their Applications, Collége de France Seminar Volume XIV, Elsevier Science B.V., 2002, pp. 533-569.

[16] Y. Maday, A. T. Patera, G. Turinici, Global a priori convergence theory for reduced-basis approximation of single-parameter symmetric coercive elliptic partial differential equations, C. R. Acad. Sci. Paris, Série I 335 (3) (2002) 289-294.

[17] Y. Maday, A. T. Patera, G. Turinici, A priori convergence theory for reducedbasis approximations of single-parameter elliptic partial differential equations, 

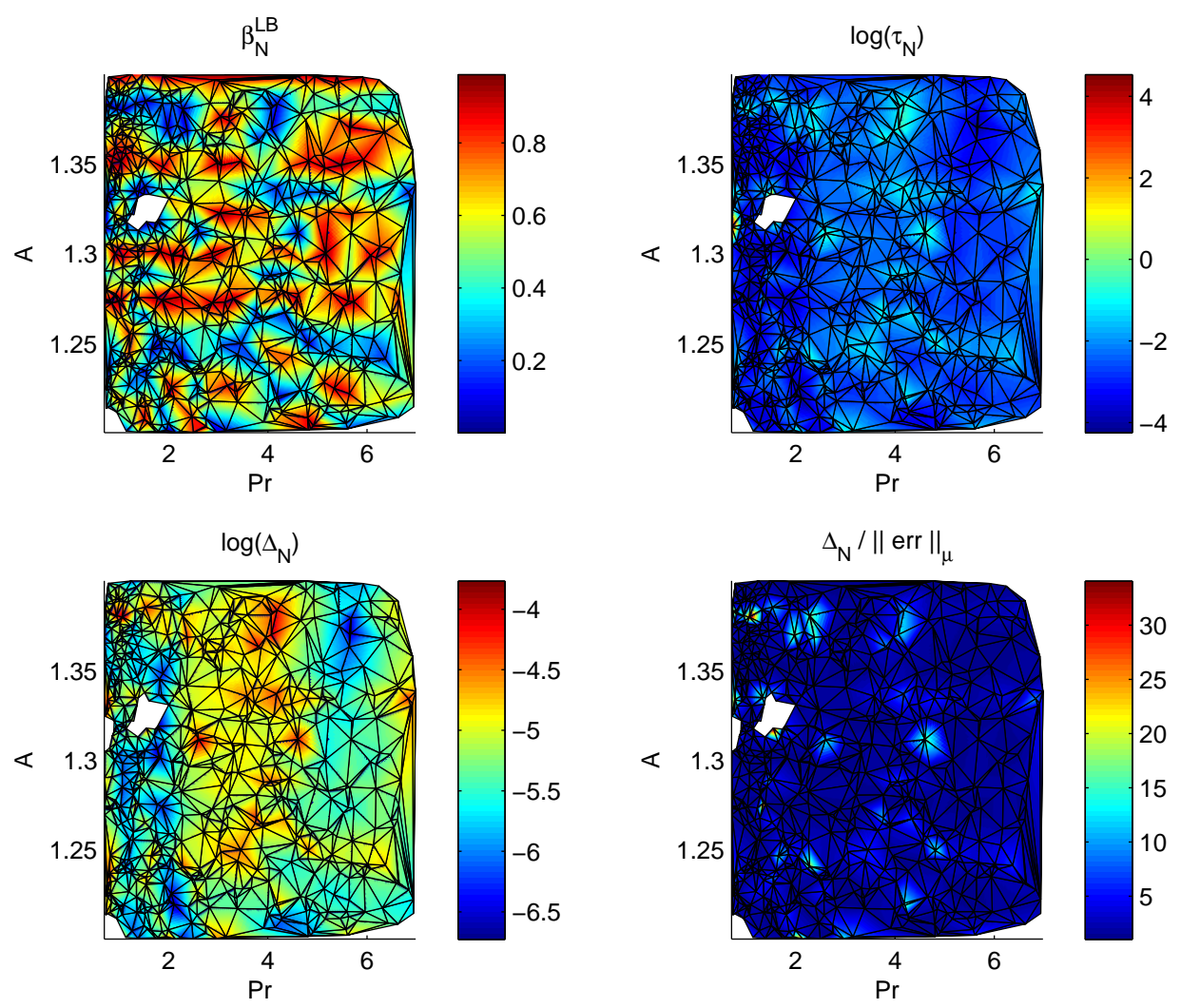

Fig. 12. One physical and one geometrical parameter: Prandtl and aspect ratio in the range $[1,7] \times[1.25,1.5]$ and $\mathcal{V}^{K}=31$. The $x$-axis represents the Grashof number and the $y$-axis the aspect ratio, both in linear scale. Upper left: inf-sup lower bound $\bar{B}_{\overline{\boldsymbol{\mu}}}^{L B}(\boldsymbol{\mu})$. Upper right: $\tau_{\overline{\boldsymbol{\mu}}}(\boldsymbol{\mu})$. Lower right: $\Delta_{N, \overline{\boldsymbol{\mu}}}(\boldsymbol{\mu})$. In the small hole we are not providing any error bound. Lower left: effectivity on the field variable error w.r.t. the natural norm.

Journal of Scientific Computing 17 (1-4) (2002) 437-446.

[18] N. C. Nguyen, G. Rozza, D. B. P. Huynh, A. T. Patera, Reduced basis approximation and a posteriori error estimation for parametrized parabolic pdes; Application to real-time Bayesian parameter estimation, in: L. Biegler, G. Biros, O. Ghattas, M. Heinkenschloss, D. Keyes, B. Mallick, L. Tenorio, B. van Bloemen Waanders, K. Willcox (eds.), Computational Methods for Large Scale Inverse Problems and Uncertainty Quantification, John Wiley and Sons, UK, 2008.

[19] N. C. Nguyen, G. Rozza, A. T. Patera, Reduced basis approximation and a posteriori error estimation for the time-dependent viscous Burgers equation, submitted.

[20] N. C. Nguyen, K. Veroy, A. T. Patera, Certified real-time solution of parametrized partial differential equations, in: S. Yip (ed.), Handbook of Materials Modeling, Springer, 2005, pp. 1523-1558.

[21] A. T. Patera, G. Rozza, Reduced Basis Approximation and A Posteriori Error Estimation for Parametrized Partial Differential Equations, Copyright 

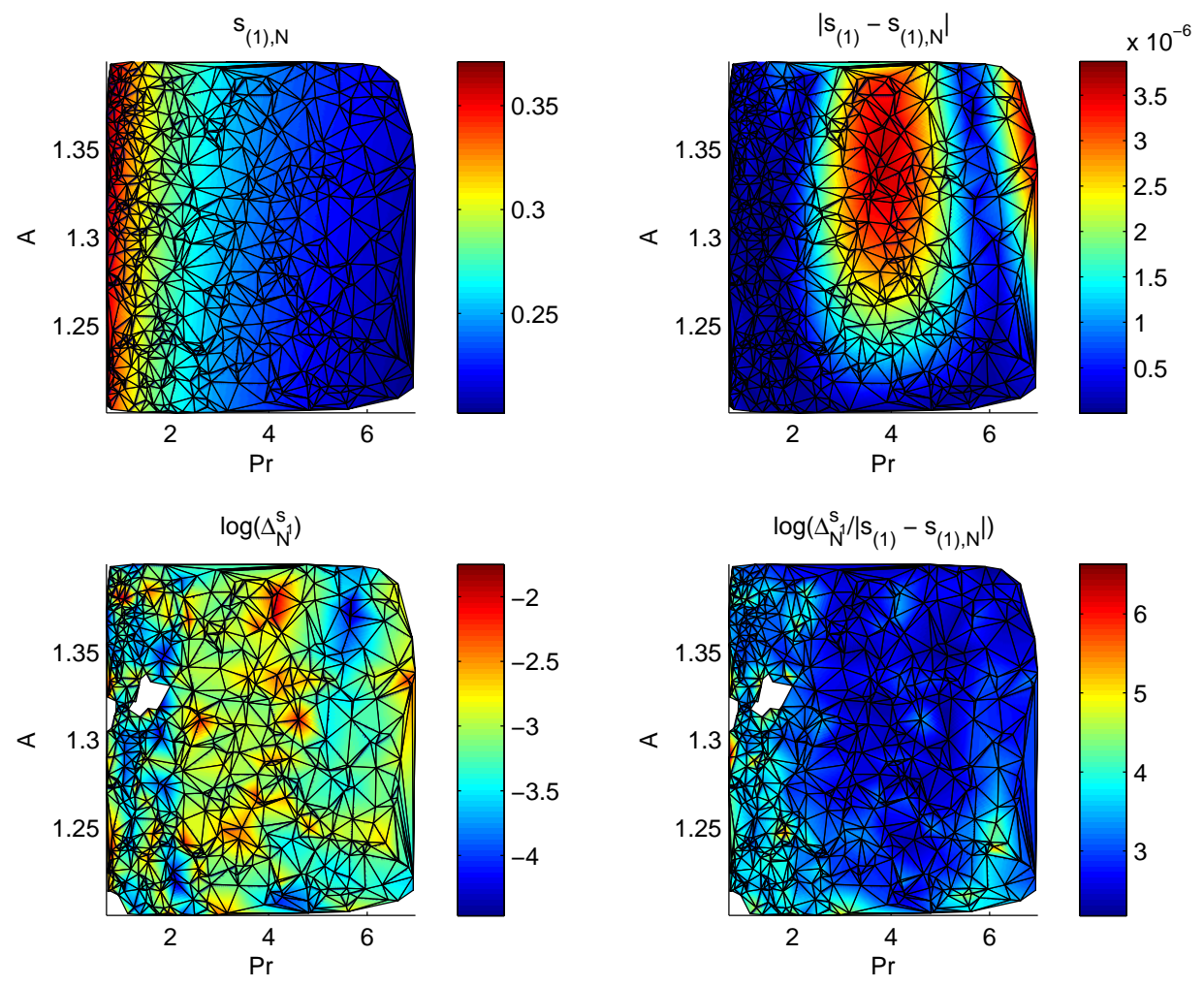

Fig. 13. One physical and one geometrical parameter: Prandtl and aspect ratio in the range $[1,7] \times[1.25,1.5]$ and $\mathcal{V}^{K}=31$. The $x$-axis represents the Prandtl number and the $y$-axis the aspect ratio, both in linear scale. Upper left: output $s_{(1)}$. Upper right: real error $\left|s_{(1)}(\boldsymbol{\mu})-s_{(1), N}(\boldsymbol{\mu})\right|$ between finite element and reduced basis solutions. Lower left: upper bounds to error for the same output, $\Delta_{N, \bar{\mu}}^{s}(\boldsymbol{\mu})$. Lower right.: effectivity of our error upper bound, $\Delta_{N, \overline{\boldsymbol{\mu}}}^{s}(\boldsymbol{\mu}) /\left|s_{(1)}(\boldsymbol{\mu})-s_{(1), N}(\boldsymbol{\mu})\right|$.

MIT, 2006-2007, to appear in MIT Pappalardo Monographs in Mechanical Engineering.

[22] J. S. Peterson, The reduced basis method for incompressible viscous flow calculations, SIAM J. Sci. Stat. Comput. 10 (4) (1989) 777-786.

[23] C. Prud'homme, D. Rovas, K. Veroy, Y. Maday, A. T. Patera, G. Turinici, Reliable real-time solution of parametrized partial differential equations: Reduced-basis output bound methods, Journal of Fluids Engineering 124 (1) (2002) 70-80.

[24] A. Quarteroni, G. Rozza, Numerical solution of parametrized Navier-Stokes equations by reduced basis methods, Numer. Methods Partial Differential Equations 23 (4) (2007) 923-948.

[25] A. Quarteroni, G. Rozza, A. Quaini, Reduced basis methods for optimal control of advection-diffusion problem, in: W. Fitzgibbon, R. Hoppe, J. Periaux, O. Pironneau, Y. Vassilevski (eds.), Advances in Numerical Mathematics, Institute of Numerical Mathematics, Russian Academy of Sciences, Moscow 
and Department of Mathematics, University of Houston, Houston, USA, 2006, pp. 193-216.

[26] G. Rozza, Optimization, control and shape design for an arterial bypass, Internat. J. Numer. Methods Fluids 47 (10-11) (2005) 1411-1419.

[27] G. Rozza, Real-time reduced basis techniques for arterial bypass geometries, in: K. Bathe (ed.), Computational Fluid and Solid Mechanics, Elsevier, 2005, pp. 1283-1287, proceedings of the Third M.I.T. Conference on Computational Fluid and Solid Mechanics, June 14-17, 2005.

[28] G. Rozza, Shape design by optimal flow control and reduced basis techniques: applications to bypass configurations in haemodynamics, Ph.D. thesis, EPFL (2005).

[29] G. Rozza, Reduced basis method for Stokes equations in domains with nonaffine parametric dependence, Computing and Visualization in Science, In press.

[30] G. Rozza, D. B. P. Huynh, A. T. Patera, Reduced basis approximation and a posteriori error estimation for affinely parametrized elliptic coercive partial differential equations: Application to transport and continuum mechanics, Archives Computational Methods in Engineering 15 (3) (2008) 229-275.

[31] G. Rozza, K. Veroy, On the stability of the reduced basis method for Stokes equations in parametrized domains, Comput. Methods Appl. Mech. Engrg. 196 (7) (2007) 1244-1260.

[32] M. Sala, Domain decomposition preconditioners: Theoretical properties, application to the compressible euler equations, parallel aspects., Ph.D. thesis, EPFL (2003).

[33] M. Sala, M. Heroux, Robust algebraic preconditioners with IFPACK 3.0, Tech. Rep. SAND-0662, Sandia National Laboratories (2005).

[34] M. Sala, K. Stanley, M. Heroux, Amesos: A set of general interfaces to sparse direct solver libraries, in: Proceedings of PARA'06 Conference, Umea, Sweden, 2006 .

[35] S. Sen, K. Veroy, D. Huynh, S. Deparis, N. Nguyen, A. Patera, "Natural norm" a posteriori error estimators for reduced basis approximations, Journal of Computational Physics 217 (1) (2006) 37-62.

[36] K. Veroy, A. T. Patera, Certified real-time solution of the parametrized steady incompressible Navier-Stokes equations; Rigorous reduced-basis a posteriori error bounds, International Journal for Numerical Methods in Fluids 47 (2005) $773-788$.

[37] K. Veroy, C. Prud'homme, A. T. Patera, Reduced-basis approximation of the viscous Burgers equation: Rigorous a posteriori error bounds, C. R. Acad. Sci. Paris, Série I 337 (9) (2003) 619-624. 
[38] K. Veroy, C. Prud'homme, D. V. Rovas, A. T. Patera, A posteriori error bounds for reduced-basis approximation of parametrized noncoercive and nonlinear elliptic partial differential equations (AIAA Paper 2003-3847), in: Proceedings of the 16th AIAA Computational Fluid Dynamics Conference, 2003. 